\title{
Synthesis of Heat-Integrated Water Allocation Networks: A Meta-Analysis of Solution Strategies and Network Features
}

\author{
Maziar Kermani *, Ivan D. Kantor (iD) and François Maréchal \\ École Polytechnique Fédérale de Lausanne (EPFL) Valais Wallis, Industrial process and energy systems \\ engineering (IPESE) group, Rue de l'Industrie 17, case postale 440, 1951 Sion, Switzerland; \\ ivan.kantor@epfl.ch (I.D.K.); francois.marechal@epfl.ch (F.M.) \\ * Correspondence: maziar.kermani@epfl.ch
}

Received: 6 April 2018; Accepted: 3 May 2018; Published: 5 May 2018

\begin{abstract}
Industries consume large quantities of energy and water in their processes which are often considered to be peripheral to the process operation. Energy is used to heat or cool water for process use; additionally, water is frequently used in production support or utility networks as steam or cooling water. This enunciates the interconnectedness of water and energy and illustrates the necessity of their simultaneous treatment to improve energy and resource efficiency in industrial processes. Since the seminal work of Savulescu and Smith in 1998 introducing a graphical approach, many authors have contributed to this field by proposing graphically- or optimization-based methodologies. The latter encourages development of mathematical superstructures encompassing all possible interconnections. While a large body of research has focused on improving the superstructure development, solution strategies to tackle such optimization problems have also received significant attention. The goal of the current article is to study the proposed methodologies with special focus on mathematical approaches, their key features and solution strategies. Following the convention of Jeżowski, solution strategies are categorized into: decomposition, sequential, simultaneous, meta-heuristics and a more novel strategy of relaxation/transformation. A detailed, feature-based review of all the main contributions has also been provided in two tables. Several gaps have been highlighted as future research directions.
\end{abstract}

Keywords: mathematical programming; superstructure optimization; solution strategy; heat-integrated mass allocation network (HIMAN); non-linear programming; heat integration; non-isothermal mixing; benchmarking; sequential; simultaneous

\section{Introduction}

This paper addresses heat-integrated water allocation networks. Due to the similarities between water and other mass streams [1] such as hydrogen networks [2], property-based networks [3], and more generally resource conservation networks, the terminology used in this paper is based on heat-integrated mass allocation network (HIMAN). This is to emphasize the fact that most of the methodologies presented in the literature, and in this paper, can be easily applied to other resources. In HIMAN problems involving water, integration of cooling water becomes especially important [4] as it should be considered in combination with process water to satisfy industrial demands. An example of this from the pulp and paper industry was presented by Suhr et al. [5]. Figure 1 illustrates typical water pathways in industrial pulp and paper plants and the strong interconnectivity among different water users. 


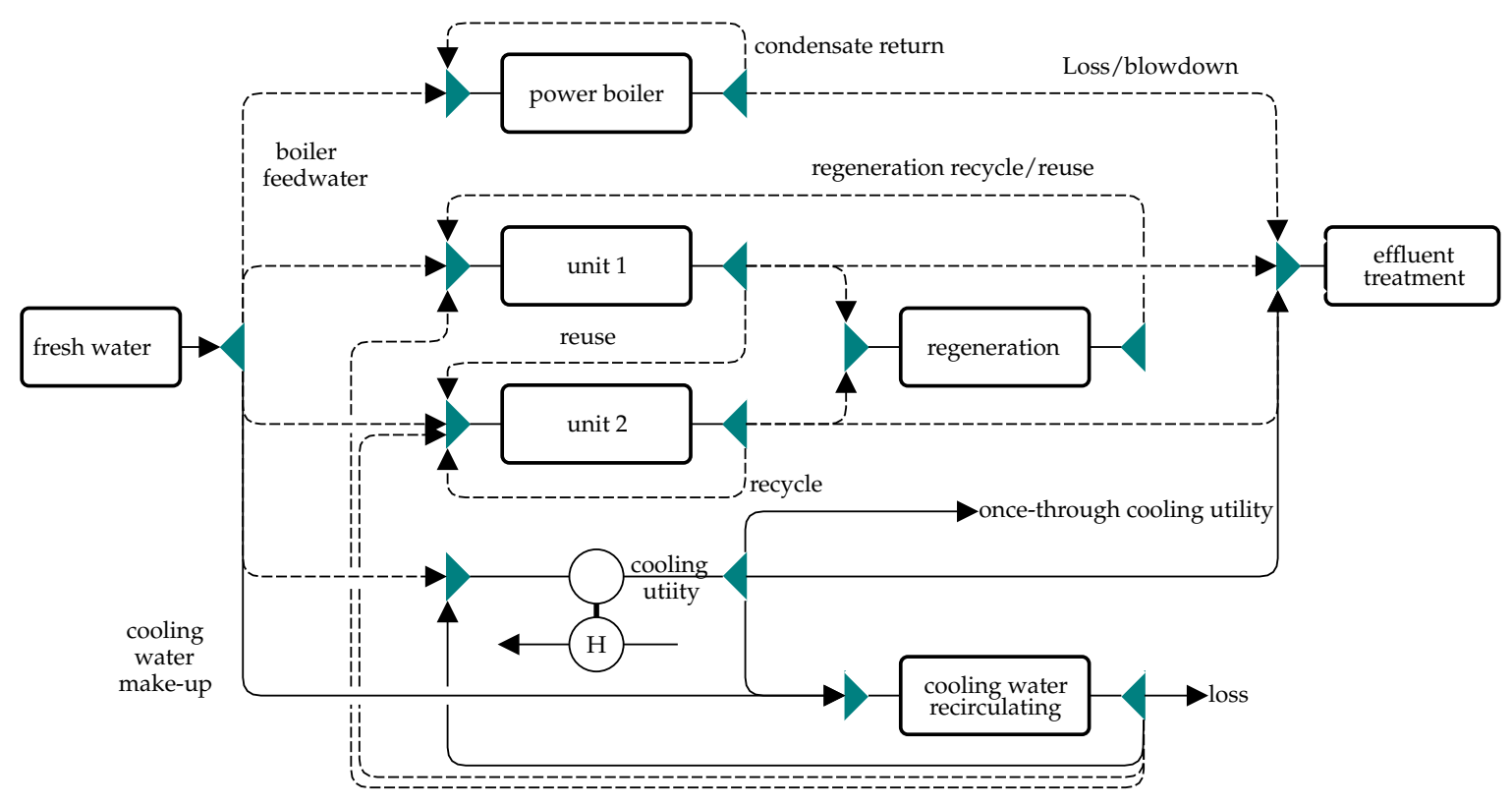

Figure 1. Schematic of water pathways in industrial plants (dash lines can be subjected to heating or cooling duties).

HIMANs have been extensively studied in the literature since their emergence in 1990 [6-8] with more than 100 articles covering different aspects and proposing various methodologies. Due to the growing interest in this domain, several review papers have been published that directly or indirectly study different features of HIMAN problems, in particular heat exchanger network (HEN) synthesis, heat-integrated water minimization, and wastewater treatment. Bagajewicz [9] provided an overview of conceptual (i.e., insight-based) and mathematical (i.e., optimization-based) methodologies in water and wastewater minimization $[10,11]$ with main focus on the authors' main contributions [12-14]. Later, Foo [15] provided a comprehensive overview of conceptual approaches in water network design (i.e., water pinch analysis) in the 21st century covering single-contaminant fixed flowrate and fixed mass load problems applied to water regeneration, treatment and total water network design. Soon after, Jeżowski [1] published an annotated exhaustive literature review on water networks, analyzing formulations, approaches, and solution strategies from 1980 to 2010 . The classification of solution strategies is modified and incorporated in the current work. Use of multi-objective optimization techniques to improve optimization and controllability of the processes together with summary of methodologies related to heat, mass and work exchange networks were provided by Chen and Wang [16]. Klemeš [17] studied recent advances in water footprinting and life cycle assessment, wastewater minimization, and heat-integrated water allocation networks. More recently, Ahmetović et al. [18] carried out a comprehensive literature review specific to HIMAN and its features covering research published until 2015. The aforementioned review papers span over 20 years of research and development; therefore, some early research directions/gaps that were highlighted could/should have already been addressed. Most of these gaps (Table 1) are addressed for mass allocation networks, however they can easily be extended to HIMANs.

The remainder of this article focuses on major features of HIMAN methodologies which are reviewed with special focus on mathematical approaches proposed after 2015. Table 2 at the end of this section provides a comprehensive overview of recent publications following the same approach employed in our previous publication [4]. For a complete review of all the related papers, the reader is referred to the published review papers $[1,9,15-18]$. 
Table 1. Highlighted gaps in the literature on water allocation networks and their specificities.

\begin{tabular}{|c|c|}
\hline Highlighted Gaps & Remarks/Literature \\
\hline Fixed concentration problems & $\begin{array}{l}\text { As opposed to fixed mass load problems in which outlet concentration } \\
\text { is limited (e.g., solubility) and hence mass load becomes variable. (not } \\
\text { extensively addressed in the literature) }\end{array}$ \\
\hline Multi-contaminant problems & $\begin{array}{l}\text { Extensively addressed by mathematical methodologies with use of nonlinear } \\
\text { programming techniques [19] }\end{array}$ \\
\hline Rigorous modeling & $\begin{array}{l}\text { Rigorous water and treatment unit models for retrofit problems in particular } \\
\text { (not extensively addressed in the literature) }\end{array}$ \\
\hline Batch-wise processes & $\begin{array}{l}\text { Seminal work by Wang and Smith [20], and several prominent works } \\
\text { covering water allocation network synthesis problem for batch processes } \\
\text { [21-27] (not extensively addressed in the literature of HIMANs) }\end{array}$ \\
\hline Non-water processes & $\begin{array}{l}\text { In particular hydrogen networks [28]. Topics on "resource conservation } \\
\text { network" and "property-based resource conservation networks" are } \\
\text { dedicated to address this particularity [29]. }\end{array}$ \\
\hline Retrofitting & $\begin{array}{l}\text { Developing methodologies for plant retrofitting considering technical } \\
\text { and geographical constraints to find feasible and practical solutions (not } \\
\text { extensively addressed in the literature). }\end{array}$ \\
\hline Uncertainty analysis & $\begin{array}{l}\text { Uncertainty and operability of water networks due to variations of flow and } \\
\text { contamination to find resilient and flexible networks [30-35] }\end{array}$ \\
\hline Multi-period operations & $\begin{array}{l}\text { Considering variations of operating condition, e.g., temperature of } \\
\text { freshwater, over multiple time horizons (to some extent, this has been } \\
\text { addressed by literature on batch-wise operations). }\end{array}$ \\
\hline Heat integration & $\begin{array}{l}\text { Extensively studied under HIMAN methodologies and is the main focus of } \\
\text { the current article. }\end{array}$ \\
\hline Interplant operations & $\begin{array}{l}\text { Extensively addressed by Chew et al. [36], Zhou et al. [37], Zhou and Li } \\
\text { [38], Ibrić et al. [39], Kermani et al. [40] in HIMAN problems. }\end{array}$ \\
\hline Improving solution strategies & $\begin{array}{l}\text { Improving deterministic approaches, application of stochastic or hybrid } \\
\text { (combined heuristic and mathematics) approaches. Jeżowski [1] highlighted } \\
\text { the use of sequential-decomposition techniques or combination of several } \\
\text { meta-heuristic (i.e., stochastic such as genetic algorithm (GA)) approaches as } \\
\text { potential directions. }\end{array}$ \\
\hline Holistic approaches [41] & $\begin{array}{l}\text { Considering synergies among different sections by extending the boundaries } \\
\text { to incorporate all aspects in an industrial plant. Several authors aimed } \\
\text { at integrating non-water thermal streams [40,42-45], cooling utilities [4], } \\
\text { and hot utilities (steam cycle) [40] in their methodologies and found that } \\
\text { application of holistic approaches can bring economical and environmental } \\
\text { benefits to all parties involved. The topic remains under-addressed in } \\
\text { the literature. }\end{array}$ \\
\hline
\end{tabular}

\section{Classification and Analysis of Key Features of Heat-Integrated Water Allocation Networks}

\subsection{Approaches}

There are two main approaches in HIMAN synthesis problems: conceptual and mathematical. Conceptual approaches make use of graphical techniques and expert insight. Several conceptual approaches have been proposed in the past with focus on single-contaminant problems including, but not limited to: two-dimensional grid diagram [6,46,47], heat surplus diagram [48], water energy balance diagram [49], superimposed mass and energy curve [50,51], temperature vs. concentration diagram [52,53], and enthalpy difference vs. flow chart approach [54]. Only two conceptual approaches (concentration order and temperature composite curve [55,56] and single-temperature-peak design principle [57]) have been proposed to handle multi-contaminant problems.

Mathematical approaches, conversely, are based on superstructure derivation and optimization which take into account many interconnection possibilities in the network design. The mathematical formulation is generally non-convex mixed integer non-linear programming (MINLP) and the objective function is mainly defined as minimization of total annualized cost (TAC) of the system, including both operating and investment cost. Solving a rigorous superstructure is very complex and hence 
requires innovative solution strategies. Mathematical approaches and their solution strategies are analyzed in more detail in Section 3.

There exists a third approach combining the synergies of conceptual and mathematical approaches. Hybrid methodologies [58-65] were first highlighted by Bagajewicz [9] as the most effective alternative to their individual applications. Such approaches allow the use of insight-based heuristics in formulating the mathematical models and hence aid mathematical approaches in representing practical and realistic alternatives in their superstructure. Moreover, expert insight can be incorporated in the methodologies to evaluate the solutions at each stage of the solution strategy, similar to the methodology proposed by Kermani et al. [4]. Conceptual approaches can also be used as techniques for initialization of large MINLP superstructures. The research direction is mainly focused on mathematical approaches. From the optimization perspective, mathematical approaches are guaranteed to provide optimal solutions (or near-optimal in non-convex formulations) to the problem, however the feasibility of such solution(s) in practice is not guaranteed. For this reason, hybrid approaches must be the main focus for future research.

\subsection{Interconnectivity of Heat and Water}

The proposed methodologies (being categorized as conceptual or mathematical approaches) can be categorized into three groups considering the interconnectivity of heat and water as "separate", "sequential", and "simultaneous". In "separate" methodologies, fresh water consumption is minimized in the first step, while the water network is designed without considering temperature constraints of the network. Knowing these two, in the second step, thermal streams will be extracted for heat integration [64,66-68]. "Sequential" methodologies are similar to "separate" methodologies in the fact that fresh water consumption is minimized in the first stage; however, this target is incorporated in the second step, where heat integration and water network design are performed simultaneously $[6,14,42,69,70]$. "Simultaneous" methodologies, on the other hand, consider all the aforementioned steps simultaneously by taking into account the trade-offs between water consumption and thermal utility consumptions.

\subsection{Water Network Specificities}

Single vs. multiple contaminants: With regard to the constituent of water streams, the problem can be formulated as single-contaminant or multi-contaminant. It should be highlighted that the research focusing on property (e.g., toxicity, viscosity, or acidity) integration in resource conservation approaches can also be categorized under this classification. The mathematical formulations dealing with contaminations are generally nonlinear due to the existence of bilinear terms of type $\dot{\mathbf{m}}_{u} \mathbf{C}_{u}$ at the inlet of mixers, where $\dot{\mathbf{m}}_{u}$ and $\mathbf{C}_{u}$ are unknown mass flowrate and contamination, respectively. Savelski and Bagajewicz [12] showed that for single-contaminant problems, the contaminant will always reach its highest limit at the outlet of a water unit operation. Therefore, the nonlinear equality constraint at the inlet of a mixer can be formulated as a linear inequality constraint with outlet contamination fixed at its maximum value. Using the necessary condition of optimality proposed by Savelski and Bagajewicz [13] and the maximum driving force [11], Yang and Grossmann [71] formulated a linear model for targeting fresh water consumption in multi-contaminant problems by relaxing the equality constraint of a mixer to an inequality constraint. The direction of relaxation was achieved by applying the KKT (Karush-Kuhn-Tucker) conditions of optimality. They stated that this formulation will result in the exact target under certain conditions and otherwise provides a tight upper bound to the problem.

Fixed-load vs. fixed-flow problems: Water minimization problem formulations can be categorized into two groups of fixed-load (FL) problems and fixed-flow (FF) problems [15,57,72]. In fixed-load problems, water is essentially a mass transfer medium with the goal of removing a fixed amount of mass load (e.g., contamination) from a process. Cleaning processes are considered as this 
type of problem. Since water can enter and leave a process $u$ at any level of contamination $\left(\mathbf{C}_{u}^{\text {in }}\right.$ and $\mathbf{C}_{u}^{\text {out }}$, respectively), water flowrate $\left(\mathbf{F}_{u}\right)$ through each process varies according to Equation (1):

$$
\mathbf{F}_{u}=\frac{\mathrm{L}_{u}}{\left(\mathbf{C}_{u}^{\text {out }}-\mathbf{C}_{u}^{\text {in }}\right)} \quad \forall u \in \mathbf{W U P}
$$

where WUP is the set of water unit processes. This type of problem implies equal flowrates at the inlet and outlet of each water unit processes. Nonetheless, water loss or gain can be modeled as well with additional modifications. The limiting composite curve approach [10] and mass problem table (similar to problem table algorithm in heat cascade) [73] are among the well-known conceptual approaches based on fixed-load problems. For fixed-flow problems, the flowrate through each process is fixed while the water unit process is modeled as two separate units, i.e., source and sink. Conversion of the fixed-load problem into a fixed-flow problem for single-contaminant processes is completed by fixing the flow to the limiting flowrate using Equation (1) (By setting $\mathbf{C}_{i}^{i n}=\mathrm{C}_{i}^{\text {in, max }}$ and $\mathbf{C}_{i}^{\text {out }}=\mathrm{C}_{i}^{\text {out } \text {,max }}$ ).

\subsection{Heat Exchanger Network Synthesis}

HEN synthesis problem is among the most researched topics in process integration dealing with developing more rigorous superstructures while providing more efficient solution strategies. An early review paper by Gundersen and Naess [74] presents more than 200 publications on this topic while Furman and Sahinidis [75] provides a comprehensive overview of major solution strategies and studies conducted in HEN synthesis until the end of the 20th century. HEN design in HIMAN synthesis problems is different from the classical HEN synthesis problems due to the possibility of stream mixing and splitting within HENs. All conceptual approaches use the classical pinch design method after having maximized the indirect heat exchanges (non-isothermal mixing (NIM)). To understand the implication of HEN synthesis in HIMAN problems using mathematical approaches, it is vital to provide a brief summary of HEN synthesis methodologies. The complete HEN superstructure is an MINLP model with nonlinear terms in both the objective function and constraints. Proposed solution strategies are directly affected by the proposed superstructure (modification of the original superstructure by relaxation, linearization, etc.) and can be categorized mainly as sequential vs. simultaneous solution strategies:

- Sequential approaches: The HEN synthesis problem can be broken down into several subproblems which is then solved successively for the minimum total HEN cost. The general approach is a three-step sequential technique. The first step minimizes the utility consumption through either conceptual techniques such as pinch design method [76] or mathematical techniques by constructing mixed integer linear programming (MILP) models [77,78] . Having the utility targets, an MILP model is formulated in the second step to minimize the number of matches between hot and cold streams which is known as the heat load distribution (HLD) problem $[77,79,80]$. This step can further be divided into subproblems for each pinch interval which effectively minimizes the number of heat exchanger units instead of matches. In the last step, a non-linear programming (NLP) model [81] can be solved for minimum cost of heat exchanger network subject to results of the two previous steps. Floudas et al. [81] showed that every solution of the second step corresponds to a feasible HEN design in the third step. Floudas and Ciric [82] proposed a decomposition solution strategy for solving the NLP model of HEN synthesis to global optimality using generalized Benders decomposition (GBD) given the HLD matches and the utility targets and a fixed heat recovery approach temperature. Nonetheless, the solutions of the second step will only provide a feasible match with minimum number of matches and cannot guarantee a globally optimum HEN in the third step. Many techniques exist to direct the second step toward better matching results. Implementing integer cut constraints [83] to generate many solutions in the second step with minimum number of matches or using penalty (i.e., ranking) costs for each match in the objective function of the second step are among 
these techniques. Spaghetti design (i.e., vertical heat transfer model) [84] can also be incorporated into an MILP model (proposed by Gundersen and Grossmann [85] and extended by Gundersen et al. [86]) for targeting and ranking matches which may result in lower capital cost.

- Simultaneous approaches: The goal is to design the HEN at once. The two major contributions in this category are the works of Floudas and Ciric [82] and Yee and Grossmann [87] which are based on MINLP modeling. The former is indeed a combination of the MILP model of Papoulias and Grossmann [77] for heat load distribution and the NLP model of Floudas et al. [81] while the latter is based on a stage-wise representation approach [88]. Several assumption are incorporated in the stage-wise approach which results in a linear set of constraints, while the nonlinearity only arises in the objective function due to logarithmic mean temperature difference formulation. However, as is discussed below, this is not the case in HIMAN due to the presence of NIM.

For mathematical approaches, the main difficulty arises in modeling the heat duty of water streams which are not known a priori. This is important as the HEN formulation is generally constructed by knowing the set of hot and cold streams in advance. Superstructure-based methodologies are often suggested to solve the problem by incorporating all possible interconnections but the computational burden for a comprehensive superstructure is often cited to be an issue [89]. To address this, a subset of water streams can be integrated with HEN. The survey of literature shows that fresh water and wastewater streams are dominantly modeled as a succession of heat exchangers and splitters, and heat exchangers and mixers, respectively. The rest of water streams, i.e., inlet streams, outlet streams, and recycling streams may or may not be included in HEN synthesis superstructure. In mathematical approaches, the dominant HEN superstructures are the modified state-wise superstructure of Yee and Grossmann [87] and the state-space superstructure of Bagajewicz et al. [90]. The former is modified by including stream splitting, stream mixing, and non-isothermal mixing options which consequently makes the problem non-convex with nonlinearities arising in both objective function and constraints. The superstructure formulation of Papoulias and Grossmann [77] has also been used by many authors $[4,58]$ to generate feasible heat exchange matches while the final HEN design is carried out using pinch design method. The HEN hyperstructure of Floudas and Ciric [82] has also been applied by Leewongtanawit and Kim [91] within a decomposition solution strategy.

\subsection{Wastewater Regeneration and Treatment}

Water regeneration implies removing impurities using treatment techniques which can later be reused or recycled in the system. Generally, regeneration units are categorized as fixed outlet concentration (provides linear models [1]) or fixed removal ratio approaches. This should not be confused with treatment units which remove impurities in disposed waste due to environmental regulations; though nevertheless, the classification remains the same. Methodologies for optimization of wastewater regeneration and treatment networks can be broadly categorized into conceptual and mathematical approaches. Conceptual approaches for wastewater treatment are limited to non-heat-integrated networks; nevertheless, a short summary of these methodologies is included here as they bring insights into optimal integration of treatment units with processes. Several conceptual techniques have been developed:

- Fixed-load problems: Early work on regeneration targeting in this category is based on limiting composite curve approaches [10,11,92]. However, as stated by Foo [15], these techniques could not handle all different cases that could arise. In particular, there are cases where implementing the regeneration process changed the pinch point [93] and hence cannot correctly define the minimum fresh water target. Later, several studies proposed using graphical and sequential approaches to overcome this issue, known as revised targeting techniques [94,95]. They showed that the inlet concentration of a regeneration unit is not always the same as the pinch concentration (assumption that was made in previous work). In each case, a fixed outlet concentration for regeneration units were considered. 
- Fixed-flowrate problems: Hallale [96] presented a guideline for placement of regeneration units in fixed-flowrate problems. Analogous to the placement of heat pumps in thermal processes, they indicated that, to reduce the fresh water intake (analogous to reducing hot utility in conventional pinch analysis), a regeneration unit should be placed across the pinch by regenerating water with higher concentration from above the pinch (having excess water, analogous to excess heat below the pinch in conventional pinch analysis) to the lower concentration region below the pinch (water deficit, analogous to heat deficit above the pinch in conventional pinch analysis). The main conceptual methods include ultimate flow targeting, source composite curve, and automated targeting techniques. Nonetheless, separate analysis of wastewater treatment networks and water networks forbids any potential reduction in fresh water consumption.

Mathematical models of wastewater treatment and regeneration are nonlinear in nature due to the existence of bilinear terms and hence resulting in non-convex NLP formulations. Quesada and Grossmann [97] highlighted two formulation approaches in modeling general multi-component problems: considering mass flow and composition components unknown which makes the mass balance constraints of mixers nonlinear, while another approach is to model the individual flows of components which makes the mass balance constraints of splitters nonlinear. The former approach is the dominant one in water allocation networks. Quesada and Grossmann [97] proposed a linearization formulation using McCormick relaxation [98] within a branch and bound procedure to solve the problem to global optimality. Karuppiah and Grossmann [19] were the first to address the advantage of optimization of integrated water networks including wastewater treatment. Similar to Quesada and Grossmann [97], they incorporated the McCormick formulation for convex relaxation of bilinear terms in the original NLP model. They later extended their superstructure to address uncertainty within contamination generation and treatment removal ratio by proposing non-convex MINLP models and solve the problem to optimality. The detailed description of mathematical techniques in solving non-convex NLP and MINLP models are beyond the scope of this review and the readers are encourage to refer to $[19,97,99-103]$.

Dong et al. [104] were the first to address total heat-integrated water allocation networks incorporating wastewater regeneration units and their interconnections within an MINLP superstructure. They showed that the combination of deterministic and stochastic search techniques typically reached the global optimum. Yang and Grossmann [71] have proposed a linear programming (LP) targeting model for water networks involving treatment units using a similar approach to the HEN stage-wise superstructure of Yee and Grossmann [87]. Their proposed model includes as many stages as the number of treatment technologies and one pathway for each unit operation, implying no mixer at the inlet of a treatment unit. The resulting targeting superstructure does not necessarily provide an upper bound, yet does provide approximations of the optimal value of the objective function for the original NLP model. Sharma and Rangaiah [67] applied the same formulation of Bogataj and Bagajewicz [105] for regeneration units while using multi-objective optimization (MOO) through a GA minimizing the total fresh water intake and total regenerated water. Besides two studies $[69,106]$ that have modeled the treatment units using the fixed outlet contamination approach, others have used the fixed removal ratio approach.

The full list of all the works in HIMANs together with their classifications and key features is presented in Table 2. 
Table 2. Literature review of methodologies on HIMAN.

\begin{tabular}{|c|c|c|c|c|c|c|c|c|c|c|c|c|c|c|c|}
\hline \multicolumn{2}{|r|}{ Legends } & \multicolumn{3}{|c|}{ Methodology } & \multicolumn{3}{|c|}{ Approach } & \multicolumn{8}{|c|}{ Energy and Mass Features } \\
\hline $\begin{array}{r}\mathrm{S} \\
\mathrm{M} \\
\mathrm{FL} \\
\mathrm{FF} \\
\mathrm{I} / \mathrm{II}\end{array}$ & $\begin{array}{l}\text { Single } \\
\text { Multiple } \\
\text { Fixed Load } \\
\text { Fixed Flow } \\
\text { Stage } 1 \text { or } 2 \text { of the } \\
\text { Methodology }\end{array}$ & \begin{tabular}{l}
$\bar{\tau}$ \\
\multirow{2}{*}{} \\
$\stackrel{0}{0}$ \\
$\tilde{0}$ \\
0
\end{tabular} & 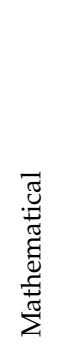 & 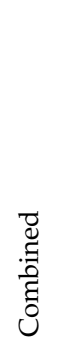 & 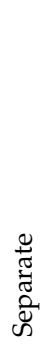 & 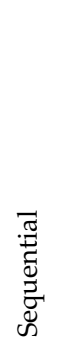 & 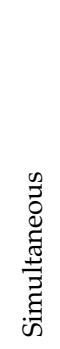 & 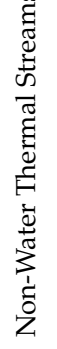 & 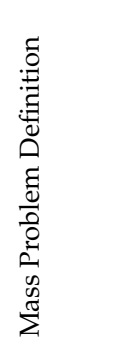 & 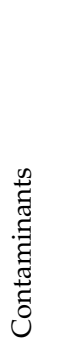 & 文 & 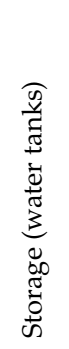 & 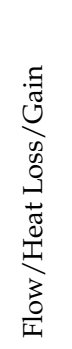 & 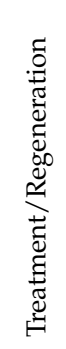 & 胥 \\
\hline Sriniva & s and El-Halwagi [8] & & - & & & & - & & FL & $\mathrm{S}$ & & & & & \\
\hline Papale & xandri and Pistikopoulos [7] & & - & & & & - & & FL & $\mathrm{S}$ & - & & & & - \\
\hline Savule & scu and Smith [6] & - & & & & - & & & FL & $\mathrm{S}$ & - & & & & - \\
\hline Bagaje & wicz et al. [14] & & & - & & $\bullet$ & & & FL & S & - & & & & - \\
\hline Savule & scu et al. [107] & - & & & & - & & & FF & $\mathrm{S}$ & - & & & & - \\
\hline Boond & arik Leewongwanawit [108] & & $\bullet$ & & & & - & & FL & S & - & & & & $\bullet$ \\
\hline Du et $c$ & 1. [42] & & - & & & - & & & FL & M & & & & & - \\
\hline Sorin a & nd Savulescu [109] & - & & & & $\bullet$ & & & FL & S & - & & & & $\bullet$ \\
\hline Savule & scu et al. [46] & - & & & & & - & & FL & $\mathrm{S}$ & - & & & & - \\
\hline Savule & scu et al. [47] & $\bullet$ & & & & & - & & FL & S & - & & - & & $\bullet$ \\
\hline Bogata & jand Bagajewicz [110] & & - & & & & - & & FL & $\mathrm{S}$ & - & & & & - \\
\hline Liao et & al. [111] & & $\bullet$ & & & - & & & FL & S & $\bullet$ & & & & $\bullet$ \\
\hline Leewo & ngtanawit and Kim [91] & & $\bullet$ & & & & - & & FF & M & - & & - & & - \\
\hline Feng e & al. [58] & & & - & & & $\bullet$ & & FL & M & $\bullet$ & & & & $\bullet$ \\
\hline Dong & $\mathrm{t}$ al. [104] & & - & & & & - & & FL & $\mathrm{M}$ & - & & & - & - \\
\hline Bogata & and Bagajewicz [105] & & - & & & & - & & FL & M & - & & & $\bullet$ & $\bullet$ \\
\hline Xiao et & al. [112] & & $\bullet$ & & & & $\bullet$ & & FL & M & $\bullet$ & & & & $\bullet$ \\
\hline Manar & et al. [48] & $\bullet$ & & & & - & & & $\mathrm{FL} / \mathrm{FF}$ & S & - & & & & $\bullet$ \\
\hline Leewo & ngtanawit and Kim [49] & $\bullet$ & & & & $\bullet$ & & & & S & $\bullet$ & & & & $\bullet$ \\
\hline Kim et & al. [113] & & - & & & & - & & FL & M & & & & & $\bullet$ \\
\hline Feng e & al. [114] & & - & & & - & & & FL & $\mathrm{S}$ & - & & & & \\
\hline Ataei $€$ & $\mathrm{t}$ al. [59] & & & - & & & - & & FL & S & - & & - & - & $\bullet$ \\
\hline Polley & et al. [66] & - & & & - & & & & FL & $\mathrm{S}$ & & & & & - \\
\hline Chen $\mathrm{C}$ & t al. [106] & & - & & & & - & & & M & - & $\bullet$ & & - & - \\
\hline Ataei a & nd Yoo [60] & & & - & & & - & & FL & M & $\bullet$ & & - & $\bullet$ & - \\
\hline Wan A & Iwi et al. [50] & - & & & & & $\bullet$ & & FL & S & $\bullet$ & & & & $\bullet$ \\
\hline Martín & ez-Patiño et al. [52] & - & & & & - & & & FL & $\mathrm{S}$ & - & - & & & - \\
\hline Ismail & et al. [51] & $\bullet$ & & & & & - & & FF & $S$ & $\bullet$ & & & & $\bullet$ \\
\hline Liao et & al. [115] & & $\bullet$ & & & & $\bullet$ & & FL & S & $\bullet$ & & & & $\bullet$ \\
\hline Georg & et al. [116] & & $\bullet$ & & & $\bullet$ & - & & FF & M & - & & & & $\bullet$ \\
\hline Bandy & padhyay and Sahu [117] & - & & & & & - & & FL & S & & & & & $\bullet$ \\
\hline Zhou & tal. [37] & & $\bullet$ & & & & $\bullet$ & & FF & M & - & & & - & $\bullet$ \\
\hline Zhou e & t al. [118] & & - & & & & - & & $\mathrm{FL} / \mathrm{FF}$ & M & - & & & - & - \\
\hline Yiqing & et al. [119] & - & & & & - & & & FL & $\mathrm{S}$ & - & & - & & $\bullet$ \\
\hline Tan et & al. $[120]$ & & $\bullet$ & & & & $\bullet$ & & FF & S & $\bullet$ & & & & $\bullet$ \\
\hline Sahu a & nd Bandyopadhyay [61] & & & - & & $\bullet$ & & & FF & M & - & & $\bullet$ & & - \\
\hline Renarc & et al. [43] & & - & & & & $\bullet$ & - & FF & S & $\bullet$ & & & & \\
\hline Martín & ez-Patiño et al. [53] & $\bullet$ & & & & & $\bullet$ & & FL & S & $\bullet$ & & & & $\bullet$ \\
\hline Boix et & al. [69] & & - & & & - & & & FL & $\mathrm{S}$ & - & & & - & - \\
\hline Ahmet & ović and Kravanja [121] & & $\bullet$ & & & I & II & & FL & M & - & & & & $\bullet$ \\
\hline Yang a & nd Grossmann [71] & & $\bullet$ & & & & $\bullet$ & & FL & M & $\bullet$ & & & $\bullet$ & \\
\hline Tan et & al. $[62]$ & & & $\bullet$ & & & - & & $\mathrm{FL} / \mathrm{FF}$ & M & - & & & & $\bullet$ \\
\hline Rojas- & orres et al. [122] & & - & & & & - & & FL & M & & & & & \\
\hline Li [123 & & & $\bullet$ & & & & $\bullet$ & & $\mathrm{N} / \mathrm{A}$ & M & - & & & & $\bullet$ \\
\hline Liu et & 1. [63] & & & - & & I & II & & FL & $\mathrm{S}$ & - & & & & - \\
\hline Ibrić e & al. [124] & & - & & & & $\bullet$ & & FL & M & - & & & & $\bullet$ \\
\hline Chew & t al. [125] & & $\bullet$ & & & & $\bullet$ & & FL & S & $\bullet$ & & & & \\
\hline Ahmet & ović and Kravanja [126] & & $\bullet$ & & & & $\bullet$ & & FL & M & - & & & & $\bullet$ \\
\hline Tan et & al. [127] & & $\bullet$ & & & & - & & FF & M & $\bullet$ & & & & $\bullet$ \\
\hline Sharm & and Rangaiah [67] & & $\bullet$ & & - & & & & FF & M & $\bullet$ & & & - & $\bullet$ \\
\hline Kerma & ni et al. [44] & & - & & & & - & - & FF & M & - & - & & & - \\
\hline Jiméne & z-Gutiérrez et al. [128] & & $\bullet$ & & & & - & & FL & M & - & & & & - \\
\hline Ibrić et & al. [129] & & $\bullet$ & & & & $\bullet$ & & FL & M & $\bullet$ & & & & $\bullet$ \\
\hline Ibrić et & al. [130] & & - & & & & $\bullet$ & & FL & $\mathrm{M}$ & - & & & $\bullet$ & $\bullet$ \\
\hline
\end{tabular}


Table 2. Cont.

\begin{tabular}{|c|c|c|c|c|c|c|c|c|c|c|c|c|c|c|c|}
\hline \multicolumn{2}{|r|}{ Legends } & \multicolumn{3}{|c|}{ Methodology } & \multicolumn{3}{|c|}{ Approach } & \multicolumn{8}{|c|}{ Energy and Mass Features } \\
\hline $\begin{array}{r}\mathrm{S} \\
\mathrm{M} \\
\mathrm{FL} \\
\mathrm{FF} \\
\mathrm{I} / \mathrm{II}\end{array}$ & $\begin{array}{l}\text { Single } \\
\text { Multiple } \\
\text { Fixed Load } \\
\text { Fixed Flow } \\
\text { Stage } 1 \text { or } 2 \text { of the } \\
\text { Methodology }\end{array}$ & 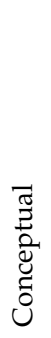 & 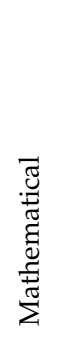 & 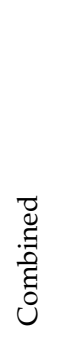 & 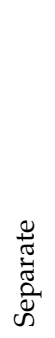 & 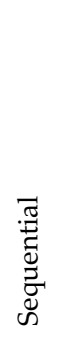 & 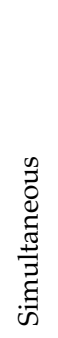 & 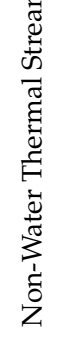 & 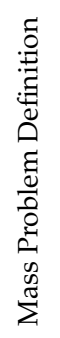 & 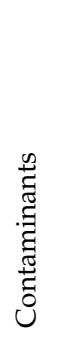 & 文 & 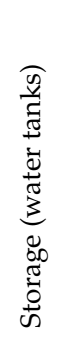 & 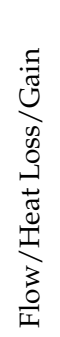 & 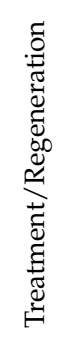 & 壼 \\
\hline \multicolumn{2}{|c|}{ Ibrić et al. [131] } & & $\bullet$ & & & & $\bullet$ & & FL & M & $\bullet$ & & & $\bullet$ & - \\
\hline \multicolumn{2}{|c|}{ Hou et al. [55] } & - & & & & & - & & FL & M & & & & & - \\
\hline \multicolumn{2}{|c|}{ Chen et al. [132] } & & - & & & & - & & FL & M & - & & & - & - \\
\hline \multicolumn{2}{|c|}{ Ahmetović and Kravanja [133] } & & - & & & & - & & FL & M & - & & & & - \\
\hline \multicolumn{2}{|c|}{ Ahmetović et al. [134] } & & - & & & & - & & FL & M & - & & & - & - \\
\hline \multirow{2}{*}{\multicolumn{2}{|c|}{$\begin{array}{l}\text { Zhou and Li [38] } \\
\text { Zhou et al. [135] }\end{array}$}} & & - & & & & - & & FL & M & - & & & - & - \\
\hline & & & - & & & & - & & FL & $\mathrm{S}$ & - & & & & - \\
\hline \multicolumn{2}{|c|}{ Zhao et al. [70] } & - & & & & - & & & FL & S & $\bullet$ & & & & $\bullet$ \\
\hline \multicolumn{2}{|c|}{$[136,137]$} & & - & & & & $\bullet$ & & FL & $S$ & $\bullet$ & & & & $\bullet$ \\
\hline \multicolumn{2}{|c|}{ Liao et al. [54] } & - & & & & & $\bullet$ & & FL & S & $\bullet$ & & & & $\bullet$ \\
\hline \multicolumn{2}{|c|}{ Ghazouani et al. [138] } & & - & & & & $\bullet$ & & FF & S & $\bullet$ & & & & \\
\hline \multicolumn{2}{|c|}{ Yan et al. [139] } & & - & & & & $\bullet$ & & FL & M & $\bullet$ & & & & $\bullet$ \\
\hline \multicolumn{2}{|c|}{ Xie et al. [57] } & - & & & & & $\bullet$ & & FF & M & $\bullet$ & & & & $\bullet$ \\
\hline \multicolumn{2}{|c|}{ Torkfar and Avami [140] } & & - & & & & - & & FL & $\mathrm{S}$ & - & & & & - \\
\hline \multicolumn{2}{|c|}{ Liang and Hui [68] } & & $\bullet$ & & - & & & & FL & S & $\bullet$ & & & & \\
\hline \multicolumn{2}{|c|}{ Jagannath and Almansoori [141] } & & $\bullet$ & & & & - & & FL & M & $\bullet$ & & & - & $\bullet$ \\
\hline \multicolumn{2}{|c|}{ Ibrić et al. [142] } & & & $\bullet$ & & & $\bullet$ & & FL & M & $\bullet$ & & & $\bullet$ & $\bullet$ \\
\hline \multicolumn{2}{|c|}{ Hong et al. [143] } & & - & & & & - & & FL & $\mathrm{S}$ & - & & & & - \\
\hline \multicolumn{2}{|c|}{ De-León Almaraz et al. [64] } & & & - & - & & & & FL & M & - & & - & - & - \\
\hline \multicolumn{2}{|c|}{ Wang et al. [65] } & & & $\bullet$ & & & - & & FL & $S$ & $\bullet$ & & & & $\bullet$ \\
\hline \multicolumn{2}{|c|}{ Kermani et al. [4] } & & & $\bullet$ & & & $\bullet$ & $\bullet$ & FF & M & $\bullet$ & - & & & $\bullet$ \\
\hline \multicolumn{2}{|c|}{ Ibrić et al. [45] } & & $\bullet$ & & & & $\bullet$ & $\bullet$ & FL & M & $\bullet$ & & & $\bullet$ & $\bullet$ \\
\hline \multicolumn{2}{|c|}{ Ghazouani et al. [144] } & & $\bullet$ & & & & $\bullet$ & & FF & M & $\bullet$ & & & & $\bullet$ \\
\hline Ibrić et & al. [39] & & - & & & & - & & FL & M & - & & & - & - \\
\hline Hong $\mathrm{e}$ & t al. [145] & & $\bullet$ & & & & $\bullet$ & & FL & S & $\bullet$ & & & & $\bullet$ \\
\hline Hong $\mathrm{e}$ & tal. [146] & & - & & & & - & & FL & M & - & & & - & - \\
\hline Hou et & al. [56] & - & & & & & - & & FL & $\mathrm{S}$ & - & & & & - \\
\hline Liu et $a$ & 1. [147] & & - & & & & $\bullet$ & & FL & M & $\bullet$ & & & - & $\bullet$ \\
\hline
\end{tabular}

\section{Superstructure Generation and Solution Strategies}

In general, the synthesis problem of HIMANs is formulated as an MINLP problem. This is due to the presence of binary variables (existence of heat or mass exchange matches) and continuous variables (operating conditions, e.g., temperature and contamination levels) which are complex to solve. This necessitates the development of robust and efficient solution strategies. Several solution strategies can be applied to HIMAN superstructures depending on the interconnectivity, complexity and completeness of the superstructure. As stated by Jeżowski [1], they can be categorized into linearization, initialization, sequential, decomposition, meta-heuristics and simultaneous techniques. Simultaneous solution strategies for solving MINLP problems may exhibit decomposition or sequential techniques intrinsic to the solver being used. They are, however, categorized under simultaneous strategies. It should also be highlighted that the categorization can overlap to some degree, i.e., several techniques can be combined in a solution strategy. Table 3 provides a feature-based representation of all mathematical approaches in HIMAN synthesis problems addressing their objective function(s), mathematical formulation and solution strategy. 
Table 3. Comparison of articles on combined water and energy integration (mathematical approaches).

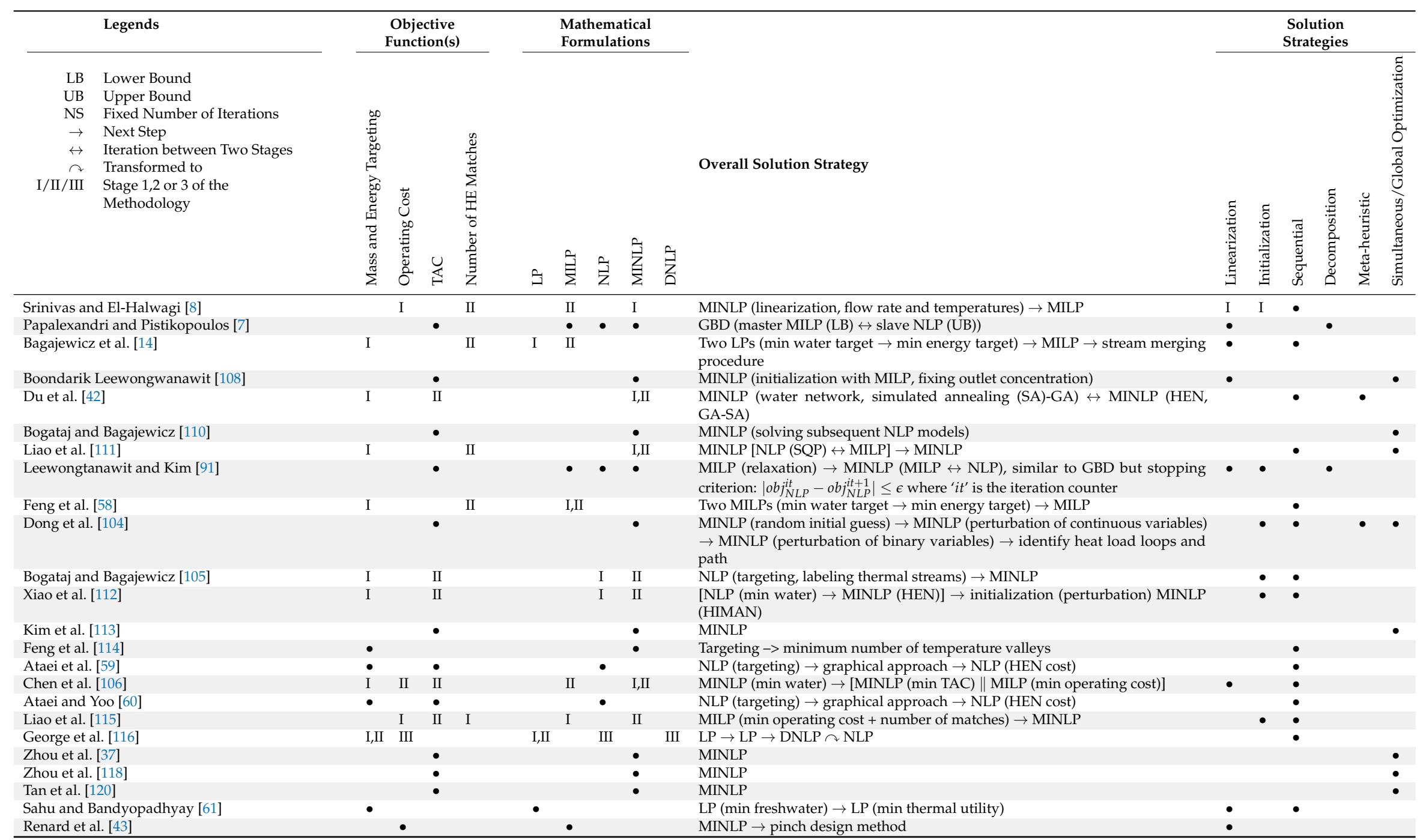


Table 3. Cont

\begin{tabular}{|c|c|c|c|c|c|c|c|c|c|c|c|c|c|c|c|}
\hline \multicolumn{2}{|r|}{ Legends } & \multicolumn{4}{|c|}{$\begin{array}{c}\text { Objective } \\
\text { Function(s) }\end{array}$} & \multicolumn{4}{|c|}{$\begin{array}{l}\text { Mathematical } \\
\text { Formulations }\end{array}$} & \multirow[b]{2}{*}{ Overall Solution Strategy } & \multicolumn{5}{|c|}{$\begin{array}{l}\text { Solution } \\
\text { Strategies }\end{array}$} \\
\hline $\begin{array}{r}\text { LB } \\
\text { UB } \\
\text { NS } \\
\rightarrow \\
\leftrightarrow \\
\stackrel{\leftrightarrow}{\curvearrowright} \\
\mathrm{I} / \mathrm{II} / \mathrm{III}\end{array}$ & $\begin{array}{l}\text { Lower Bound } \\
\text { Upper Bound } \\
\text { Fixed Number of Iterations } \\
\text { Next Step } \\
\text { Iteration between Two Stages } \\
\text { Transformed to } \\
\text { Stage } 1,2 \text { or } 3 \text { of the } \\
\text { Methodology }\end{array}$ & 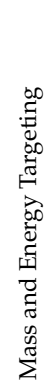 & 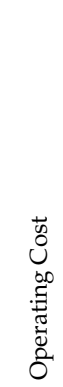 & U্জ & 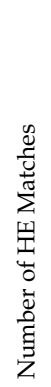 & G & $\stackrel{\vec{B}}{\mathrm{~B}}$ & $\vec{z}$ & $\stackrel{\vec{z}}{\vec{z}}$ & & 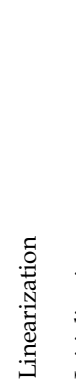 & 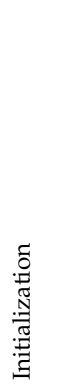 & 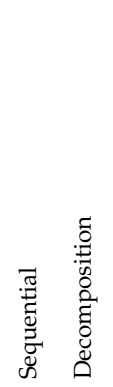 & 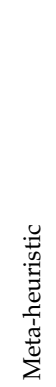 & 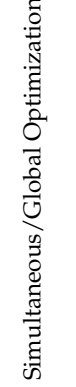 \\
\hline \multicolumn{2}{|c|}{ Boix et al. [69] } & I & & II & & & I & & II & $\begin{array}{l}\text { MILP [ } \epsilon \text {-constraint MOO] (min freshwater, thermal utility, number of water } \\
\text { connections, and number of thermal matches) } \rightarrow \text { MINLP }\end{array}$ & • & & $\bullet$ & & • \\
\hline \multicolumn{2}{|c|}{ Ahmetović and Kravanja [121] } & I & & II & & & & • & - & Solving water network (NLP, MINLP) initialization $\rightarrow$ MINLP (HIMAN) & & & I & & II \\
\hline \multicolumn{2}{|c|}{$\begin{array}{l}\text { Yang and Grossmann [71] } \\
\text { Tan et al. [62] }\end{array}$} & - & & & & - & & • & & Targeting & • & & - & & \\
\hline \multicolumn{2}{|c|}{ Tan et al. [62] } & & $\bullet$ & $\bullet$ & & & $\bullet$ & & $\bullet$ & $\begin{array}{l}\text { MINLP discretization to MILP (use of floating pinch concept to identify role of } \\
\text { thermal streams, i.e., hot or cold) }\end{array}$ & $\bullet$ & & & & - \\
\hline \multicolumn{2}{|c|}{ Rojas-Torres et al. [122] } & & & - & & & & & - & MINLP & & & & & - \\
\hline \multicolumn{2}{|c|}{ Li [123] } & I & & II & & & & I & II & Particle swarm optimization (NLP $\rightarrow$ MINLP) & & & - & • & \\
\hline \multicolumn{2}{|c|}{ Liu et al. [63] } & I & & II & & & & & II & GA-SA: I) mass pinch + pseudo-T-H diagram $\rightarrow$ II) MINLP & & & - & - & \\
\hline \multirow{2}{*}{\multicolumn{2}{|c|}{$\begin{array}{l}\text { Ibrić et al. [124] } \\
\text { Chew et al. [125] }\end{array}$}} & & I & II & & & & I & II & NLP (UB for utilities) $\rightarrow$ MINLP & & I & - & & II \\
\hline & & & I & II & & & & I & II & NLP (operating cost) $\rightarrow$ MINLP (min TAC) & & & & & - \\
\hline \multicolumn{2}{|c|}{$\begin{array}{l}\text { Chew et al. [125] } \\
\text { Ahmetovic and Kravania [126] }\end{array}$} & & & • & & & & & • & MINLP & & • & & & • \\
\hline \multicolumn{2}{|c|}{ Tan et al. [127] } & & - & & & & & & - & MINLP & & & & & - \\
\hline \multicolumn{2}{|c|}{ Sharma and Rangaiah [67] } & $\bullet$ & & & & & & & $\bullet$ & $\begin{array}{l}\text { MINLP }[\epsilon \text {-constraint MOO] (min freshwater, regenerated water) } \rightarrow \text { pinch design } \\
\text { method }\end{array}$ & & & $\bullet$ & & \\
\hline \multicolumn{2}{|c|}{ Kermani et al. [44] } & • & & & & & • & & & MILP $\rightarrow$ pinch design method & & & - & & \\
\hline \multicolumn{2}{|c|}{ Jiménez-Gutiérrez et al. [128] } & & & • & & & & & • & MINLP & & & & & - \\
\hline \multirow{2}{*}{\multicolumn{2}{|c|}{ Ibrić et al. [129] }} & & I & II & & & & I & II & NLP (UB for utilities) $\rightarrow$ MINLP & & • & & & - \\
\hline & Ibrić et al. [130] & & I & II & & & & I & II & Same as Ibrić et al. [129] + wastewater treatment & & • & & & • \\
\hline \multicolumn{2}{|c|}{ Ibrić et al. [131] } & & I & II & & & & I & II & Same as Ibrić et al. [129] + wastewater treatment + multi-choice splitting & & • & & & - \\
\hline \multicolumn{2}{|c|}{ Chen et al. [132] } & & & - & & & & & - & MINLP & & & & & • \\
\hline \multicolumn{2}{|c|}{ Ahmetović and Kravanja [133] } & & & - & & & & & • & MINLP (considering heat integration for recycled and reused water streams) & & & & & - \\
\hline \multicolumn{2}{|c|}{ Ahmetović et al. [134] } & & & - & & & & & - & Same as Ahmetović and Kravanja [133] + wastewater treatment & & & & & - \\
\hline \multicolumn{2}{|c|}{ Zhou and $\mathrm{Li}[38]$} & & & - & & & & & • & $\begin{array}{l}\text { Local optimum (MINLP } \leftrightarrow \text { relaxed-MINLP perturbation) } \rightarrow \text { clustering } \\
\text { technique }\end{array}$ & & $\bullet$ & $\bullet$ & $\bullet$ & \\
\hline \multicolumn{2}{|c|}{ Zhou et al. [135] } & & & $\bullet$ & & & & & $\bullet$ & $\begin{array}{l}\text { MINLP } \curvearrowright \text { mathematical programming with equilibrium constraints (MPEC) } \curvearrowright \\
\text { NLP }\end{array}$ & & & & & $\bullet$ \\
\hline \multicolumn{2}{|c|}{ Liu et al. [136,137] } & & & • & & & & & • & Generalized disjunctive programming $(\mathrm{GDP}) \curvearrowright \mathrm{MINLP}$ & & & - & & \\
\hline Ghazouan & i et al. [138] & & - & & & & • & & & MILP & & & • & & \\
\hline Yan et al. [ & & & & $\bullet$ & & & & • & & NLP (relaxing integers with fractional continuous variables) & & & & & $\bullet$ \\
\hline Torkfar an & d Avami [140] & & & - & & & & & - & MINLP (including pressure drops in water network) & & & & & N/A \\
\hline
\end{tabular}


Table 3. Cont.

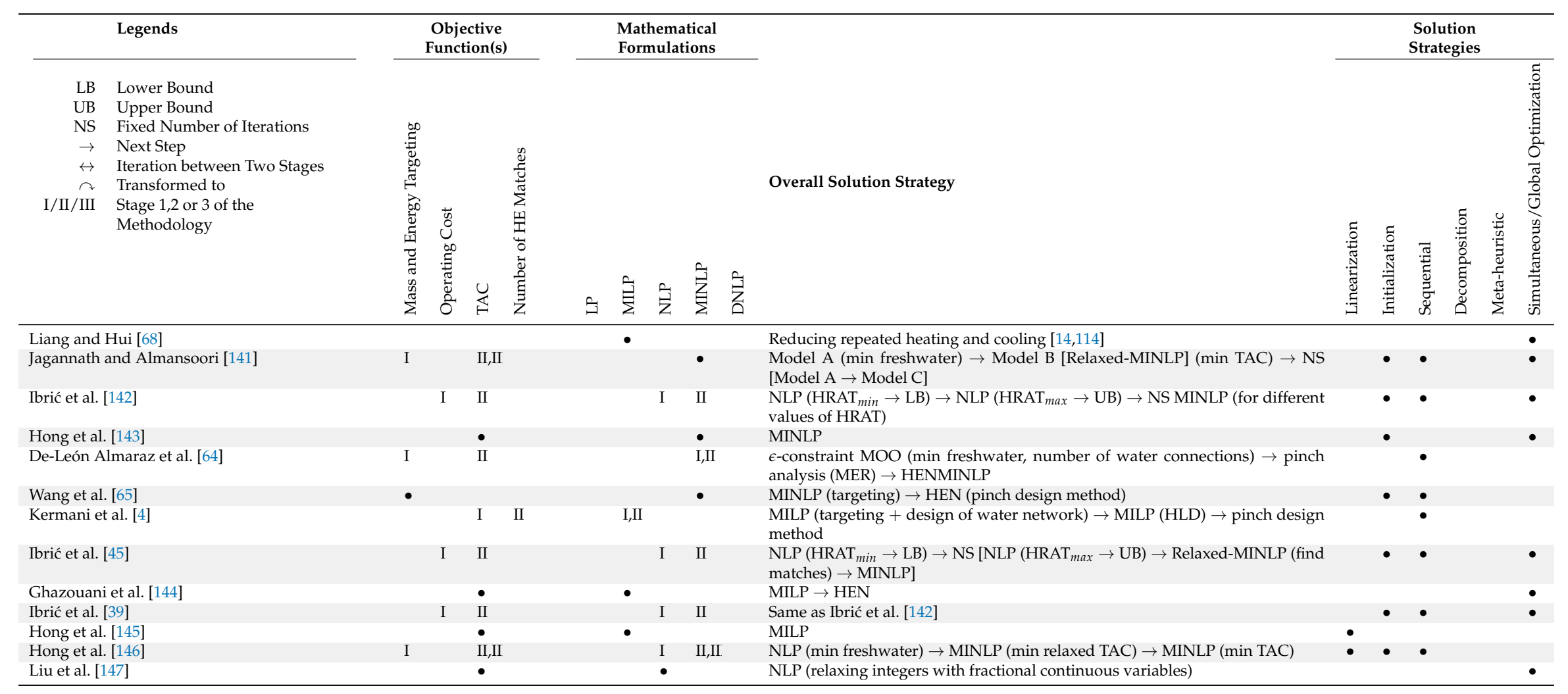




\subsection{Decomposition}

Two terminologies of "decomposition" and "sequential" must be clarified here. In both cases, the problem is divided into two or more steps; however, the former consists of a finite number of iterations between the steps given defined termination criteria, while the latter is a uni-directional solution strategy with no iteration. More importantly, in decomposition solution strategies (such as GBD) the results of one step provide inputs for the subsequent steps while in sequential solution strategies, no such interactions exist. Papalexandri and Pistikopoulos [7] proposed an MINLP superstructure for heat and mass exchange networks and solved it using GBD [148] by decomposing the problem into a master MILP model optimizing the network configuration and a primal NLP model optimizing the operating conditions. The MILP model provided a non-decreasing lower bound to the objective function while the NLP model gave a non-increasing upper bound. The stopping criterion was the convergence of the objective functions in the two steps below a predefined threshold. They have allocated the binary and continuous variables to master and primal problems, respectively. Similarly, Leewongtanawit and Kim [91] decomposed their MINLP model into MILP and NLP models and solved them iteratively until no further improvement (beyond a threshold) was observed in the objective function of the NLP model. They decomposed the variables similar to the approach proposed by Floudas and Ciric [82] in HEN synthesis.

\subsection{Sequential}

Several sequential solution strategies have been proposed which commonly optimize water and heat targets within the first or second step using LP [14,61], MILP $[4,44,58]$, or NLP $[59,60]$ models. Having these targets (with or without the design of water network), an MILP model can be formulated to minimize the number of heat exchange matches $[77,79]$. In all cases, the HEN is designed using the pinch design method. Liao et al. [111] proposed a two-step approach for targeting and design by formulating two MINLP models. The first model obtained the water and thermal utility targets with number of stream splits, while the second model minimized the number of heat exchange matches. Dong et al. [104] proposed an iterative sequential solution strategy by first solving an MINLP model using random initial guesses and later improving the results (solving the MINLP model at each step) by iterative heuristic perturbations in both continuous and binary variables. Liao et al. [115] solved an MILP model minimizing the operating cost together with number of matches (similar to HLD) and used the targets and matches as initialization for the MINLP model of HIMAN. Most recently, Ibrić et al. [142] proposed an iterative sequential solution strategy consisting of two steps. An NLP water network model was solved for minimum heat recovery approach temperature (HRAT) to provide a lower bound on the problem. In the second step, an upper bound is assigned to HRAT using a finite number of iterations and a sequence of NLP-MINLP models were solved for each value with the final solution selected as the best among all solutions. Jagannath and Almansoori [141] proposed a sequential solution strategy by introducing three MINLP models: Model A (water network with NIM), Model C (HEN synthesis [87]) and Model B (combined Models A and C). The problem was solved sequentially by solving Model A and a simplified version of Model A to find the water and energy targets. Depending on the results of the two versions, a relaxed version of Model B was solved. At this point, the flow and concentration variables in Model A were fixed and the problem was solved to generate set of solutions using techniques similar to integer cuts. Model $C$ was applied for each solution and the final optimal solution was thus selected as the best of all solutions. The authors mentioned that their sequential approach is computationally exhaustive, yet the solutions are similar to those obtained by simultaneous approaches. More recently, Hong et al. [146] extended their targeting approach [145] by addressing multi-contaminant as well was treatment problems using a sequential solution strategy. An NLP model was formulated to minimize the fresh water consumption in a first step, providing initial values on flow rates and concentrations. An MINLP model was then solved with relaxed/linear TAC in the second step followed by optimization of the original MINLP in the third step. 


\subsection{Simultaneous with or Without Initialization}

For simultaneous approaches, models are mainly formulated as MINLP which are solved using commercial software such as DICOPT [149], BARON [150,151], SBB or LINDO [152]. It should be noted that the algorithms underlying these solvers (e.g., outer approximation [153] in DICOPT) are based on decomposition techniques. Nevertheless the original mathematical formulation was not decomposed before solving $[110,113,120,143])$. Generally, an MINLP model requires good initialization which can be achieved by solving a relaxed instance of the original model either through fixing continuous variables (resulting in an MILP initialization model), fixing binary variables (mainly by excluding the HEN matches, and hence resulting in an NLP model [124,126,129-131,134]), solving an MINLP model of water network with no heat integration [133], or by generating random values [38,135]. Boondarik Leewongwanawit [108] used an MILP model for initialization of MINLP superstructure by fixing the contamination loading at the outlet of water units and linearizing the HEN cost formulation. Dong et al. [104] used an NLP formulation of the water network for targeting the utility consumption and further labeling thermal streams (i.e., hot or cold) which was then solved simultaneously.

\subsection{Meta-Heuristics}

Du et al. [42] have employed GA combined with SA for optimizing water network (MINLP superstructure) and HEN (MINLP superstructure) in an iterative manner. They, however, neglected temperature effects in the water superstructure (first step) and hence construct (i.e., extract) the required thermal streams based on the optimized water network for the second step. Liu et al. [63] proposed a hybrid methodology combining GA-SA with mass pinch and pseudo-T-H-diagram [154]. Other meta-heuristics approaches such as particle swarm optimization were incorporated in the work of Li [123].

\subsection{Relaxation/Transformation}

Under this classification, the original MINLP model is transformed/simplified/relaxed by redefining/removing/adding extra constraints. Zhou et al. [135] formulated a HIMAN superstructure using MPEC and applied complementarity formulations [155] to model binary variables. They solved the problem by transforming the model into NLP. GDP has been used by Liu et al. [137] to formulate discrete and continuous variables of HIMAN by incorporating logical propositions, disjunctions and algebraic constraints. They solved the problem using BARON by transforming the GDP model into MINLP. However, their formulation does not address HEN design. Several authors [139,147] proposed HIMAN superstructures using NLP techniques. Yan et al. [139] adapted the MINLP superstructure of Ahmetović and Kravanja [126] and avoided the use of binary variables by introducing continuous variables in the form of $y=f /(f+\zeta)$ where $y$ indicates the existence of the unit of size $f$ and $\zeta$ is a very small parameter $\left(\sim 10^{-5} f\right)$. Nevertheless, the superstructure a priori treated fresh water and wastewater streams as the only cold and hot streams, respectively, involved in heat integration. More recently, Hong et al. [145] proposed a targeting approach using an MILP formulation in which the HEN was designed in a single step. They adapted the HEN transshipment model [77,87] addressing stream splitting and NIM within the HEN superstructure. One other possibility is by using a discretization approach in which known variables are discretized into a set of known values which will result in an MILP model [62,156]. Several authors have highlighted the challenges in solving medium and large MINLP problems and proposed a reduction strategy and a reduced superstructure to solve heat-integrated water allocation networks more easily [65,142]. Wang et al. [65] proposed several heuristics related to contamination monotonicity (only applicable to single-contaminant problems) and pinch principles together with rational NIM in order to simplify the superstructure of Liu et al. [136]. Ibrić et al. [142] also applied several rules to eliminate infeasible and impractical variables and connections from the superstructure. They showed that these simplifications can reduce the computational efforts and hence increase the solving efficiency. 
In summary, it was observed that several major superstructures have been used/proposed by previous authors for synthesizing HIMAN problems, addressed specific regions of the solution space and required specific solution strategies. One approach was the HEN hyperstructure formulation of Floudas and Ciric [82] combined with the water allocation network problem such as in Leewongtanawit and Kim [91], which was solved via a decomposition strategy. Alternatively, Papalexandri and Pistikopoulos [7] proposed a hyperstructure for heat and mass allocation networks by integrating the HEN hyperstructure [82] and an analogous mass exchange hyperstructure [157] to construct the HIMAN hyperstructure. This approach encompassed all possible interactions between the two, where each stream could be split and directed to all heat and mass exchangers while bypass streams were also included. This comprehensive model was formulated as an MINLP problem and solved using GBD. The stage-wise HEN superstructure of Yee and Grossmann [87] was the major formulation used in literature. As discussed in Section 2.4, the major assumption of the original formulation is the isothermal mixing of streams which consequently forbids many promising alternatives in the HIMAN synthesis problem. This was modified by many authors to address non-isothermal mixing and splitting. This superstructure and the one proposed by Floudas and Ciric [82] ("simultaneous match-network optimization") are categorized under simultaneous approaches. Their usage in HIMAN synthesis problems requires additional assumptions and simplifications (e.g., not considering all water stream participating in heat exchange) in the hope of alleviating the computational burden of solving the superstructure of all possible opportunities. As an alternative to superstructure representation, the state-space representation of Bagajewicz et al. [90] was addressed in synthesizing HIMANs by several authors $[37,38,104,112,118]$. The state-space representation contains the superstructure representation of HIMAN as a special case. Nonetheless, as stated by Bagajewicz et al. [90], this representation can alleviate some of the difficulties arising in superstructure optimization. The representation is based on the definition of a set of input and output variables (e.g., input and output temperatures of thermal streams) and their relations. A state variable, such as heat exchanger inlet temperature or mass exchanger inlet concentration, is defined as a variable which allows calculation of the output variables by relation with the inputs. The state-space is referred to as the set over which the state variables take their values. To this end, the overall input-output relations can be solved via two operators: one dealing with mixing and splitting, and the other with mass or heat exchange. It was shown that use of a particular operator-the assignment operator-can lead to NLP formulations which are better suited for solving large-scale problems.

\section{Other Features}

\subsection{Superstructure Extension}

Despite the importance of holistic approaches to capture the trade-offs between heat and water, a limited amount of research has included non-water thermal streams in their methodologies. This potential was first addressed by Renard et al. [43], although no case study was presented. Later, Kermani et al. [44] extended their superstructure and presented a simplified Kraft mill process by incorporating non-water thermal streams and showed the large potential in their combination. This has been later applied to a real Kraft mill [4,40], also addressing interplant operations. Zhou et al. [37,118] developed a multi-scale, stage-wise superstructure addressing interplant operations for fixed-load as well as fixed-flowrate problems using MINLP. Most recently, Ibrić et al. [39] extended their superstructure [142] to address interplant operations by use of additional binary parameters to allow or forbid interplant connections. Heat transfer coefficient calculations have also been considered in the work of Torkfar and Avami [140] as a function of stream velocity. They further included pressure drop calculations in HEN and water network design. Their superstructure was formulated using MINLP which is stated as a modified and improved version of the superstructure by Jiménez-Gutiérrez et al. [128]. Use of live steam was first investigated by Savulescu and Smith [6]. They argued that use of live steam in water networks is doubly beneficial since it can reduce the steam 
consumption and also eliminate the use of heaters, hence reducing the capital cost. To the best of the authors' knowledge, the methodology presented by De-León Almaraz et al. [64] is the only work considering the use of vapor-state water in the water network. For implementation reasons, they modeled the phase change by only considering the sensible heat in the HEN design, while the latent heat is added to the solution via addition of a corresponding hot utility.

\subsection{Physical Improvements}

Importance of NIM and its effects on network performance were extensively highlighted throughout the literature [58,107,119,158]. Recently, Martinez-Patiño et al. [159] analyzed direct and indirect heat exchanges by incorporating an exergy component. They concluded that networks having the same water and energy targets may exhibit different exergy losses (due to NIM) which negatively impact the cost of heat exchanger area.

Features of non-water thermal streams and use of live steam in water networks must be studied further as they represent a more realistic approach for application of HIMAN methodologies in industrial applications.

\subsection{Water-Energy Nexus}

Heat-integrated water allocation network synthesis problems have been treated as a separate research field over the past decades; however, they should be regarded as a special case in the field research related to the water-energy nexus which, in turn, is part of a broader water-energy-food nexus. The water-energy nexus was first mentioned in an annual review paper by Gleick [160], highlighting the interconnectedness of energy and water. The reasoning followed that water is used in extracting and producing fuels and producing electricity via steam while energy is used to produce, transport, and purify water. In addition to the research discussed in this paper, work in water-energy nexus domain encompasses developments in the field of desalination technologies, membrane systems, water use in biorefineries, and in shale gas production. The water-energy-food nexus considers the intertwined nature of water, energy, and food by highlighting that water is used to produce food and varieties of crops which, in turn, can be used to produce biofuels. Garcia and You [161] highlighted several research opportunities related to the water-energy nexus: energy and water use in households, novel water sources such as rainwater or water being produced from extraction of fossil fuels, hydropower plants, climate studies, policy planning, and holistic approaches in design of industrial wastewater treatment networks. For comprehensive review of recent contributions and future directions related to the water-energy nexus, the reader is encouraged to refer to review papers by Garcia and You [161], Martinez-Hernandez and Samsatli [162], Lee et al. [163], Albrecht et al. [164], and Dai et al. [165].

\section{Benchmarking Analysis}

Similar to the previous review on HIMANs [18], a benchmarking analysis is carried out to illustrate the main features of different methodologies. A well-known single-contaminant case study (Table 4) originally proposed by Savulescu and Smith [6] was selected. Over 35 articles have evaluated their proposed methodologies using this case study. The results are provided in Table 5 while selected key features are plotted in Figure 2 using parallel coordinates for 33 out of 35 articles (two of them lack data to be visualized).

Several network indicators have been selected for the analysis: Number of thermal streams including thermal utilities $\left(\mathrm{N}_{s}^{t h}\right)$, number of heat exchangers $\left(\mathrm{N}_{H E}\right)$, total area of heat exchangers $\left(\mathrm{A}_{H E N}^{\text {total }}\right)$, total number of mixing points $\left(\mathrm{N}_{\text {mixer }}\right)$, number of non-isothermal mixing points $\left(\mathrm{N}_{\text {mixer }}^{N I M}\right)$, and number of mass streams in the water network excluding the thermal ones $\left(\mathrm{N}_{s}^{m}\right)$ are among them. Figure 2 shows that the number of non-isothermal mixing points as well as number of mass streams $\left(\mathrm{N}_{s}^{m}\right)$ are inversely proportional to the HEN cost, i.e., increasing either of the two will decrease the HEN cost. 
This case study further illustrates the necessity of considering more indicators while optimizing heat-integrated water allocation networks. Among the 33 visualized results in Figure 2, 29 results possess $3-5$ heat exchangers (the maximum is 10 ). This may indicate good compromise in HEN investment costs; however, for the same solutions, the number of mixers, non-isothermal mixers and total heat exchange area vary 3-13, 0-10, and 3500-6300 $\mathrm{m}^{2}$, respectively. These "neglected" indicators should be somehow addressed in HIMAN synthesis problems which necessitate the application of multi-criteria decision making approaches. In addition, no single solution can possess the optimal value for all indicators, which correspondingly requires the generation of a set of potential solutions that can be analyzed, instead of a single "optimal" solution.

Table 4. Operating data of the benchmarking test case [47].

\begin{tabular}{cccccc}
\hline Units & Mass load $\left(L_{u}\right)(\mathrm{g} / \mathrm{s})$ & $C_{u}^{\text {in, } \max }(\mathrm{ppm})$ & $C_{u}^{\text {out }, \text { in }}(\mathrm{ppm})$ & $T_{u}\left({ }^{\circ} \mathrm{C}\right)$ & Limiting Flowrate $(\mathrm{kg} / \mathrm{s})$ \\
\hline$u_{1}$ & 2 & 0 & 100 & 40 & 20 \\
$u_{2}$ & 5 & 50 & 100 & 100 & 100 \\
$u_{3}$ & 30 & 50 & 800 & 75 & 40 \\
$u_{4}$ & 4 & 400 & 800 & 50 & 10 \\
\hline
\end{tabular}

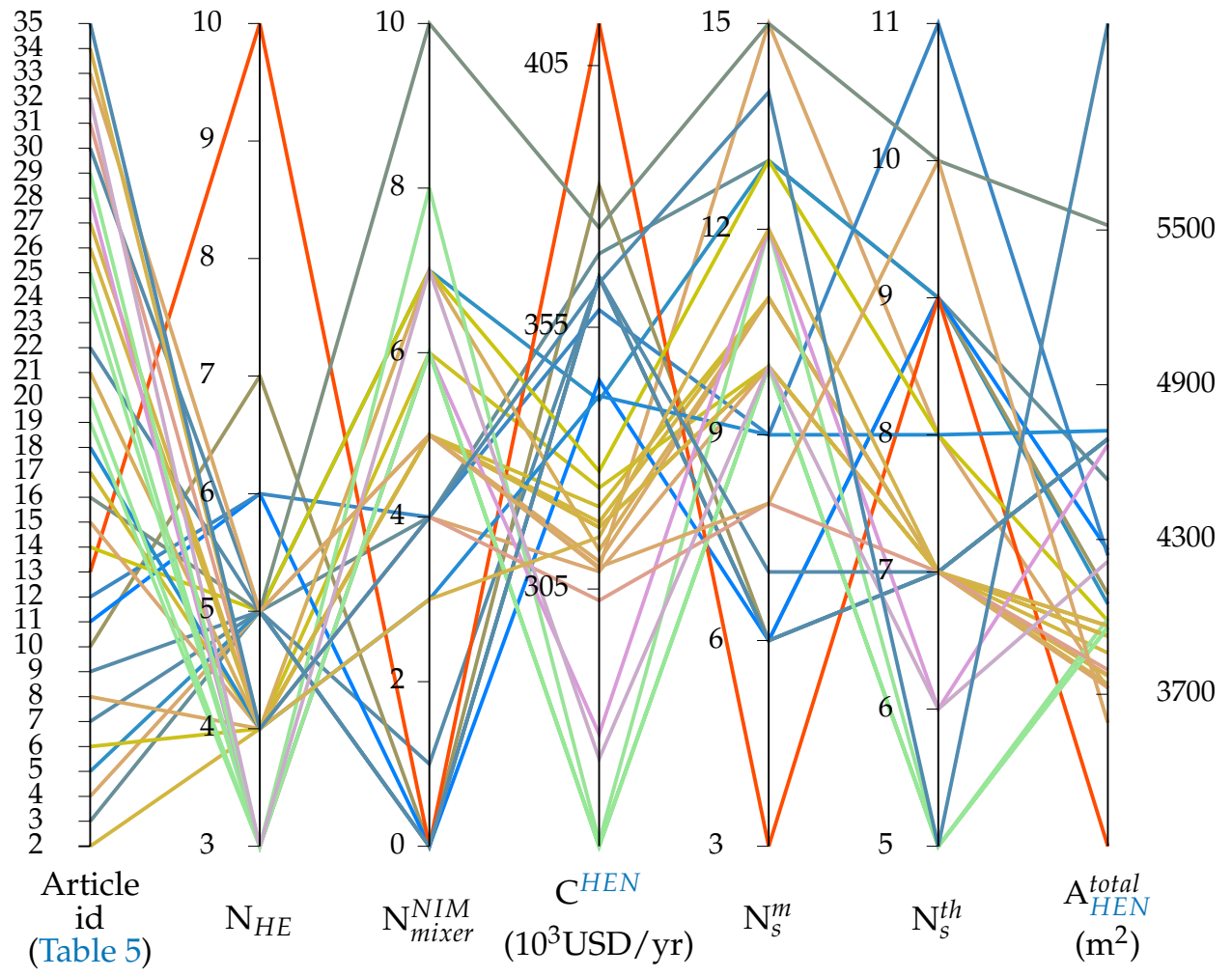

Figure 2. Visualization of some key indicators of the benchmarking case study (Table 5) using parallel coordinates [166]. 
Table 5. Benchmarking of several HIMAN methodologies using the four-water-process test case of Savulescu and Smith [6] ${ }^{(1)}$.

\begin{tabular}{|c|c|c|c|c|c|c|c|c|c|c|c|c|}
\hline \multirow[t]{2}{*}{ ID } & & & \multirow[b]{2}{*}{$\mathbf{M} / \mathbf{C}^{(2)}$} & \multicolumn{6}{|c|}{ Network Indicators ${ }^{(3)}$} & \multicolumn{2}{|c|}{ Economic Indicators ${ }^{(4)}$} & \multirow[b]{2}{*}{ Comments } \\
\hline & & & & $\mathrm{N}_{s}^{\text {th }}$ & $\mathbf{N}_{H E}$ & $\mathrm{~A}_{H E N}^{\text {total }}\left(\mathrm{m}^{2}\right)$ & $\mathrm{N}_{\text {mixer }}\left(\mathrm{N}_{\text {mimer }}^{N \text { IM }}\right)$ & $\mathrm{N}_{s}^{m}$ & $\mathrm{Q}_{H E N}^{\text {total }}(\mathrm{kW})$ & $\mathrm{C}^{\mathrm{HEN}}(\mathrm{USD} / \mathrm{yr})$ & $\mathrm{C}^{T A C}(\mathrm{USD} / \mathrm{yr})$ & \\
\hline 1 & \multicolumn{2}{|c|}{ Savulescu and Smith [6] } & $\mathrm{C}$ & 9 & 5 & NA & $10(6)$ & 15 & NA & NA & NA & \\
\hline 2 & \multicolumn{2}{|c|}{ Bagajewicz et al. [14] } & M & 7 & 4 & 3860.2 & $7(5)$ & 11 & 22,008 & 317,798 & $2,714,858$ & \\
\hline 3 & \multicolumn{2}{|c|}{ Savulescu et al. [47] } & $\mathrm{C}$ & 9 & 5 & 4530.9 & $9(4)$ & 13 & 23,585 & 369,042 & $3,040,612$ & \\
\hline 4 & \multicolumn{2}{|c|}{ Bogataj and Bagajewicz [110] } & M & 8 & 5 & 3722.1 & $7(5)$ & 15 & 22,006 & 308,889 & $2,705,949$ & Water network identical to Bagajewicz et al. [14]. \\
\hline 5 & & M & 9 & 5 & 4049.6 & $10(7)$ & 13 & 22,680 & 341,044 & $2,738,104$ & \\
\hline 6 & \multicolumn{2}{|c|}{$\begin{array}{l}\text { Dong et al. [104] } \\
\text { Bogatai and Bagaiewicz [105] }\end{array}$} & M & 7 & 4 & 3771.1 & $7(6)$ & 10 & 21,943 & 324,338 & $2,721,398$ & \\
\hline 7 & \multicolumn{2}{|c|}{ Xiao et al. [112] } & M & 7 & 5 & 4689.7 & $3(1)$ & 6 & 26,040 & 364,587 & $2,761,647$ & \\
\hline 8 & \multicolumn{2}{|c|}{ Leewongtanawit and Kim [49] } & $\mathrm{C}$ & 7 & 4 & 3775.4 & $8(5)$ & 11 & 22,260 & 310,283 & $2,707,343$ & \\
\hline 9 & \multirow[t]{3}{*}{ Polley et al. [66] } & option 1 & $\mathrm{C}$ & 7 & 5 & 4689.6 & $3(0)$ & 6 & 26,040 & 364,573 & $2,761,633$ & \\
\hline 10 & & option 2 & & 9 & 7 & 4087.8 & $4(0)$ & 6 & 23,100 & 382,059 & $2,779,119$ & \\
\hline 11 & & option 3 & & 9 & 6 & 4258.4 & $4(0)$ & 6 & 23,940 & 344,905 & $2,741,965$ & \\
\hline 12 & \multicolumn{2}{|l|}{ Mao et al. [167] } & $\mathrm{C}$ & 11 & 6 & 4238.1 & $9(4)$ & 9 & 24,071 & 358,328 & $2,755,388$ & \\
\hline 13 & \multicolumn{2}{|c|}{ Wan Alwi et al. [50] } & $\mathrm{C}$ & 9 & 10 & 3111.0 & $6(0)$ & 3 & 26,040 & 413,022 & $2,810,082$ & Infeasible HEN design (Figure 9 of ref. [50]) \\
\hline 14 & \multicolumn{2}{|c|}{ Martínez-Patiño et al. [52] } & $\mathrm{C}$ & 8 & 5 & 3984.9 & $9(7)$ & 13 & 23,527 & 327,484 & $2,954,324$ & \\
\hline 15 & \multirow{2}{*}{\multicolumn{2}{|c|}{$\begin{array}{ll}\text { Liao et al. [115] case a } & \text { case b }\end{array}$}} & M & 7 & 4 & 3725.8 & $7(4)$ & 10 & 22,008 & 308,356 & $2,705,416$ & Wastewater streams are merged \\
\hline 16 & & & & 10 & 5 & 5517.5 & $13(10)$ & 15 & 25,101 & 374,010 & $2,771,070$ & Wastewater streams are treated individually \\
\hline 17 & \multicolumn{2}{|l|}{ Yiging et al. [119] } & $\mathrm{C}$ & 7 & 4 & 3964.4 & $8(5)$ & 11 & 22,260 & 320,716 & $2,717,776$ & The best solution among two. \\
\hline 18 & \multicolumn{2}{|c|}{ Martínez-Patiño et al. [53] } & $\mathrm{C}$ & 8 & 4 & $4,721.6$ & $5(3)$ & 9 & 26,040 & 341,915 & $2,738,975$ & \\
\hline 19 & \multicolumn{2}{|c|}{ Ibrić et al. [124] } & M & 5 & 3 & 3960.1 & $6(6)$ & 10 & 22,344 & 255,873 & $2,652,933$ & Best solution with HRAT $=9^{\circ} \mathrm{C}$ \\
\hline 20 & \multicolumn{2}{|c|}{ Ahmetović and Kravanja [126] } & $\mathrm{M}$ & 5 & 3 & 3960.1 & $6(6)$ & 10 & 22,344 & 255,873 & $2,652,933$ & Same as Ibrić et al. [124] \\
\hline 21 & \multicolumn{2}{|c|}{ Liu et al. [136] } & $\mathrm{M}$ & 7 & 4 & 3739.9 & $7(7)$ & 11 & 22,008 & 312,440 & $2,709,500$ & \\
\hline 22 & \multicolumn{2}{|l|}{ Hou et al. [55] } & $\mathrm{C}$ & 7 & 5 & 4689.7 & $3(0)$ & 6 & 26,040 & 364,587 & $2,761,647$ & Polley et al. [66], option 1 \\
\hline 23 & Chen et al. [132] & & M & 9 & 12 & $\mathrm{NA}$ & NA & NA & 26,062 & NA & $\mathrm{NA}$ & \\
\hline 24 & Zhou et al. [135] & MPEC & $\mathrm{M}$ & 5 & 3 & 3960.8 & $6(6)$ & 10 & 22,344 & 255,891 & $2,652,951$ & Same as Ibrić et al. [124] \\
\hline 25 & & MINLP & & 5 & 3 & 3993.1 & $8(8)$ & 12 & 22,362 & 256,779 & $2,653,839$ & \\
\hline 26 & Zhao et al. [70] & & $\mathrm{C}$ & 7 & 4 & 3925.7 & $7(5)$ & 10 & 22,260 & 316,801 & $2,713,861$ & \\
\hline 27 & Liu et al. [137] & & M & 7 & 4 & 3925.7 & $7(5)$ & 10 & 22,260 & 316,801 & $2,713,861$ & \\
\hline 28 & Liao et al. [54] & & $\mathrm{C}$ & 6 & 3 & 4666.8 & $8(6)$ & 12 & 22,008 & 277,286 & $2,674,346$ & Case 5 - Figure 17-b of ref. [54] \\
\hline 29 & Yan et al. [139] & & M & 5 & 3 & 3960.1 & $6(6)$ & 10 & 22,344 & 255,873 & $2,652,933$ & Same as Ibrić et al. [124] \\
\hline 30 & Xie et al. [57] & & $\mathrm{C}$ & 7 & 5 & 4689.7 & $4(0)$ & 7 & 26,040 & 364,587 & $2,761,647$ & \\
\hline 31 & Torkfar and Avan & & M & 7 & 4 & 3794.6 & $5(4)$ & 8 & 22,008 & 302,830 & $2,699,890$ & \\
\hline 32 & Hong et al. [143] & case a & M & 6 & 3 & 4215.3 & $7(7)$ & 10 & 21,000 & 272,580 & $2,669,640$ & Wastewater streams are merged \\
\hline 33 & & case b & & 10 & 5 & 3589.1 & $6(5)$ & 8 & 21,807 & 309,197 & $2,706,257$ & Wastewater streams are treated individually \\
\hline 34 & Hou et al. [56] & & $\mathrm{C}$ & 7 & 4 & 3965.6 & $5(3)$ & 12 & 22,260 & 314,939 & $2,711,999$ & \\
\hline 35 & Kermani et al. [4] & & $\mathrm{M}$ & 5 & 4 & 6300.0 & $10(4)$ & 14 & 23,012 & 363,449 & $2,760,509$ & $\begin{array}{l}\text { Best solution with HRAT }=4^{\circ} \mathrm{C} \\
\text { HEN synthesis using NLP formulation [81] }\end{array}$ \\
\hline
\end{tabular}

(1) All methodologies reached the fresh water target of $90 \mathrm{~kg} / \mathrm{s}$. All but two of them reached the thermal utility targets of $3780 \mathrm{~kW}$ of hot utility. Savulescu et al. [47] and Martínez-Patiño et al. [52] reported 485 and $406 \mathrm{~kW}$ of cold utility and 4265 and $4186 \mathrm{~kW}$ of hot utility, respectively. Where information was not enough to calculate the indicators, "NA" is indicated.

2) " $\mathrm{M}$ " indicates mathematical approach, while " $\mathrm{C}$ " denotes conceptual approach.

(3) Network indicators are number of thermal streams including thermal utilities $\left(\mathrm{N}_{s}^{\text {th }}\right)$, number of heat exchangers $\left(\mathrm{N}_{H E}\right)$, total area of heat exchangers $\left(\mathrm{A}_{H E N}^{\text {total }}\right)$, total number of mixing points $\left(\mathrm{N}_{\text {mixer }}\right)$, number of non-isothermal mixing points $\left(\mathrm{N}_{m i x e r}^{N I M}\right)$, number of mass streams in the water network excluding the thermal ones $\left(\mathrm{N}_{s}^{m}\right)$, and total heat load of all the heat exchangers $\left(\mathrm{Q}_{H E N}^{\text {total }}\right)$.

(4) Economic indicators are HEN cost $\left(C^{H E N}\right)$ and total annualized cost $\left(C^{T A C}\right)$ which includes operating costs and HEN cost. 


\section{Concluding Remarks and Future Directions}

This work presented a meta-analysis of literature on heat-integrated water allocation networks. Key features of the proposed methodologies have been analyzed with special focus on mathematical programming approaches including HEN synthesis. Developing more rigorous mathematical superstructures necessitates proposing novel solution strategies. The proposed solution strategies have been categorized into decomposition, sequential, simultaneous (with or without initialization), meta-heuristics, and relaxation/transformation strategies. A benchmarking analysis was presented comparing the results of different proposed methodologies from the water and heat exchanger networks perspective. It illustrated how methodologies can produce different results considering other indicators than the typical TAC. Following this review, several gaps have been identified (Table 6 summarizes the main gaps):

- As mentioned previously, despite the importance of addressing synergies among various elements in a typical industrial plant, holistic approaches have rarely been addressed in HIMAN synthesis problems. Apart from a limited number of specific publications [4,40,43-45], non-water thermal streams have not been combined in HIMAN synthesis. Future research directions should therefore focus on this aspect by proposing more rigorous and efficient superstructures. In addition, use of live steam should be investigated using improved formulations.

- As water is subject to heating and cooling duties, water loops have a role in recovering heat within and between processes. This feature is even more sensible when considering inter-plant operations. Moreover, following the observed gap in holistic approaches, HIMAN synthesis problems should be considered in conjunction with other heat recovery technologies including organic Rankine cycles (ORC)s and heat pumps.

- The literature lacks multi-period operations of HIMANs. This is an important feature considering daily and seasonal variations of operating conditions of an industrial plant, including the temperature of freshwater. Thermal storage tanks must be combined within HIMAN problems to provide a flexible heat transfer medium over time.

- Uncertainty analysis of HIMANs must be addressed to find resilient networks given the uncertainties in the system including costs and operating conditions.

- Following the benchmarking analysis, multi-criteria decision making approaches must be incorporated in HIMAN synthesis problems to find sets of promising optimal or near-optimal solutions considering diverse economic, environmental, and practical indicators. The application of stochastic optimization and hybrid approaches should be favored in this direction.

- Upon the survey of the literature, only one article mentioned large-scale industrial applications [4], yet the methodology is limited to the targeting step. Most of the proposed mathematical methodologies are highly complex and their applications to industrial cases may face computational challenges. Hence, research toward efficient solution strategies must be the future trend thus shifting the focus toward reaching practical and good solutions, not necessarily the global optimum.

- Following the highlighted gaps in Table 1, batch processes and retrofitting remain largely untreated which necessitates further research. 
Table 6. Summary of identified gap in HIMAN synthesis problem.

\begin{tabular}{|c|c|}
\hline Gap & Description/Remarks \\
\hline \multicolumn{2}{|l|}{ Unaddressed literature gaps } \\
\hline Fixed concentration problems & Problems with variable mass load \\
\hline Rigorous modeling & Water and waste treatment models \\
\hline Multi-period operation & Considering the dynamic nature of systems \\
\hline Retrofitting & Methods covering partial system retrofit and redesign instead of design \\
\hline \multicolumn{2}{|l|}{ Newly identified gaps } \\
\hline $\begin{array}{l}\text { Better treatment of thermal } \\
\text { streams }\end{array}$ & $\begin{array}{l}\text { Considering non-water thermal streams and potential for live steam as part } \\
\text { of the problem definition }\end{array}$ \\
\hline Utility integration & Considering HIMAN with utility selection and integration concepts \\
\hline Sensitivity analysis & Generation of multiple or resilient solutions in lieu of global optima \\
\hline Multi-criteria optimization & $\begin{array}{l}\text { Methods which address multiple criteria for decision-making which extend } \\
\text { beyond minimization of cost or fresh water consumption, considering an } \\
\text { expanded system. }\end{array}$ \\
\hline Large-scale problems & $\begin{array}{l}\text { Developing approaches to adapt formulations to larger scale problems } \\
\text { or reformulation to encourage solution generation for problems on the } \\
\text { industrial scale }\end{array}$ \\
\hline
\end{tabular}

Author Contributions: This study was done as part of M.K.'s doctoral studies supervised by F.M. I.D.K. has contributed to this paper through reviewing, editing, and presentation and discussion of results.

Acknowledgments: This research project is financially supported by the Swiss Innovation Agency Innosuisse and is part of the Swiss Competence Center for Energy Research SCCER EIP.

Conflicts of Interest: The authors declare no conflict of interest.

\section{Abbreviations}

The following abbreviations are used in this manuscript:

$\begin{array}{ll}\text { GA } & \text { genetic algorithm } \\ \text { GBD } & \text { generalized Benders decomposition } \\ \text { GDP } & \text { Generalized disjunctive programming } \\ \text { HEN } & \text { heat exchanger network } \\ \text { HIMAN } & \text { heat-integrated mass allocation network } \\ \text { HLD } & \text { heat load distribution } \\ \text { HRAT } & \text { heat recovery approach temperature } \\ \text { LP } & \text { linear programming } \\ \text { MILP } & \text { mixed integer linear programming } \\ \text { MINLP } & \text { mixed integer non-linear programming } \\ \text { MOO } & \text { multi-objective optimization } \\ \text { MPEC } & \text { mathematical programming with equilibrium constraints } \\ \text { NIM } & \text { non-isothermal mixing } \\ \text { NLP } & \text { non-linear programming } \\ \text { NPV } & \text { net present value } \\ \text { SA } & \text { simulated annealing } \\ \text { TAC } & \text { total annualized cost }\end{array}$

\section{References}

1. Jeżowski, J. Review of Water Network Design Methods with Literature Annotations. Ind. Eng. Chem. Res. 2010, 49, 4475-4516. doi:10.1021/ie901632w. [CrossRef]

2. Towler, G.P.; Mann, R.; Serriere, A.J.; Gabaude, C.M.D. Refinery Hydrogen Management -Cost Analysis of Chemically Integrated Facilities. Ind. Eng. Chem. Res. 1996, 35, 2378-2388. doi:10.1021/ie950359+. [CrossRef] 
3. Shelley, M.D.; El-Halwagi, M.M. Component-less design of recovery and allocation systems: A functionality-based clustering approach. Comput. Chem. Eng. 2000, 24, 2081-2091. doi:10.1016/S0098-1354(00)00578-0. [CrossRef]

4. Kermani, M.; Périn-Levasseur, Z.; Benali, M.; Savulescu, L.; Maréchal, F. A novel MILP approach for simultaneous optimization of water and energy: Application to a Canadian softwood Kraft pulping mill. Comput. Chem. Eng. 2017, doi:10.1016/j.compchemeng.2016.11.043. [CrossRef]

5. Suhr, M.; Klein, G.; Kourti, I.; Gonzalo, M.R.; Santonja, G.G.; Roudier, S.; Sancho, L.D. Best Available Techniques (BAT) Reference Document for the Production of Pulp, Paper and Board; JRC Science and Policy Reports, Industrial Emissions Directive 2010/75/EU Integrated Pollution Prevention and Control; EU Science Hub: Brussels, Belgium, 2015.

6. Savulescu, L.E.; Smith, R. Simultaneous energy and water minimisation. In Proceedings of the 1998 AIChE Annual Meeting, 1998; pp. 13-22. Unpublished work.

7. Papalexandri, K.P.; Pistikopoulos, E.N. A multiperiod MINLP model for the synthesis of flexible heat and mass exchange networks. Comput. Chem. Eng. 1994, 18, 1125-1139. doi:10.1016/0098-1354(94)E0022-F. [CrossRef]

8. Srinivas, B.K.; El-Halwagi, M.M. Synthesis of combined heat and reactive mass-exchange networks. Chem. Eng. Sci. 1994, 49, 2059-2074. doi:10.1016/0009-2509(94)E0016-J. [CrossRef]

9. Bagajewicz, M. A review of recent design procedures for water networks in refineries and process plants. Comput. Chem. Eng. 2000, 24, 2093-2113. [CrossRef]

10. Wang, Y.P.; Smith, R. Wastewater minimisation. Chem. Eng. Sci. 1994, 49, 981-1006. doi:10.1016/0009-2509(94)80006-5. [CrossRef]

11. Wang, Y.; Smith, R. Wastewater minimization with flowrate constraints. Chem. Eng. Res. Des. 1995, 73, 889-904.

12. Savelski, M.J.; Bagajewicz, M.J. On the optimality conditions of water utilization systems in process plants with single contaminants. Chem. Eng. Sci. 2000, 55, 5035-5048. doi:10.1016/S0009-2509(00)00127-5. [CrossRef]

13. Savelski, M.; Bagajewicz, M. On the necessary conditions of optimality of water utilization systems in process plants with multiple contaminants. Chem. Eng. Sci. 2003, 58, 5349-5362. doi:10.1016/j.ces.2003.09.004. [CrossRef]

14. Bagajewicz, M.; Rodera, H.; Savelski, M. Energy efficient water utilization systems in process plants. Comput. Chem. Eng. 2002, 26, 59-79. doi:10.1016/S0098-1354(01)00751-7. [CrossRef]

15. Foo, D.C.Y. State-of-the-Art Review of Pinch Analysis Techniques for Water Network Synthesis. Ind. Eng. Chem. Res. 2009, 48, 5125-5159. doi:10.1021/ie801264c. [CrossRef]

16. Chen, Z.; Wang, J. Heat, mass, and work exchange networks. Front. Chem. Sci. Eng. 2012, 6, 484-502. doi:10.1007/s11705-012-1221-5. [CrossRef]

17. Klemeš, J.J. Industrial water recycle/reuse. Curr. Opin. Chem. Eng. 2012, 1, $238-245$. doi:10.1016/j.coche.2012.03.010. [CrossRef]

18. Ahmetović, E.; Ibrić, N.; Kravanja, Z.; Grossmann, I.E. Water and energy integration: A comprehensive literature review of non-isothermal water network synthesis. Comput. Chem. Eng. 2015, 82, 144-171. doi:10.1016/j.compchemeng.2015.06.011. [CrossRef]

19. Karuppiah, R.; Grossmann, I.E. Global optimization for the synthesis of integrated water systems in chemical processes. Comput. Chem. Eng. 2006, 30, 650-673. doi:10.1016/j.compchemeng.2005.11.005. [CrossRef]

20. Wang, Y.; Smith, R. Time Pinch Analysis. Chem. Eng. Res. Des. 1995, 73, 905-914.

21. Kim, J.K.; Smith, R. Automated Design of Discontinuous Water Systems. Process Saf. Environ. Prot. 2004, 82, 238-248. doi:10.1205/095758204323066000. [CrossRef]

22. Foo, D.C.Y.; Manan, Z.A.; Tan, Y.L. Synthesis of maximum water recovery network for batch process systems. J. Clean. Prod. 2005, 13, 1381-1394. doi:10.1016/j.jclepro.2005.04.013. [CrossRef]

23. Majozi, T. Wastewater minimisation using central reusable water storage in batch plants. Comput. Chem. Eng. 2005, 29, 1631-1646. doi:10.1016/j.compchemeng.2005.01.003. [CrossRef]

24. Cheng, K.F.; Chang, C.T. Integrated Water Network Designs for Batch Processes. Ind. Eng. Chem. Res. 2007, 46, 1241-1253. doi:10.1021/ie0611150. [CrossRef] 
25. Li, L.J.; Zhou, R.J.; Dong, H.G. State-Time-Space Superstructure-Based MINLP Formulation for Batch Water-Allocation Network Design. Ind. Eng. Chem. Res. 2010, 49, 236-251. doi:10.1021/ie900427b. [CrossRef]

26. Zhou, R.J.; Li, L.J.; Xiao, W.; Dong, H.G. Simultaneous optimization of batch process schedules and water-allocation network. Comput. Chem. Eng. 2009, 33, 1153-1168. doi:10.1016/j.compchemeng.2008.11.008. [CrossRef]

27. Chaturvedi, N.D.; Bandyopadhyay, S. Optimization of Multiple Freshwater Resources in a Flexible-Schedule Batch Water Network. Ind. Eng. Chem. Res. 2014, 53, 5996-6005. doi:10.1021/ie403638v. [CrossRef]

28. Liu, G.; Li, H.; Feng, X.; Deng, C. Novel method for targeting the optimal purification feed flow rate of hydrogen network with purification reuse/recycle. AIChE J. 2013, 59, 1964-1980. doi:10.1002/aic.13962. [CrossRef]

29. El-Halwagi, M.M. 13 - Conserving Material Resources through Process Integration: Material Conservation Networks A2 - Klemeš, Jiří J. In Handbook of Process Integration (PI); Woodhead Publishing Series in Energy; Woodhead Publishing: Sawston, UK, 2013; pp. 422-439.

30. Pistikopoulos, E.N. Uncertainty in process design and operations. Comput. Chem. Eng. 1995, 19 (Suppl. 1), 553-563. doi:10.1016/0098-1354(95)87094-6. [CrossRef]

31. Straub, D.A.; Grossmann, I.E. Design optimization of stochastic flexibility. Comput. Chem. Eng. 1993, 17, 339-354. doi:10.1016/0098-1354(93)80025-I. [CrossRef]

32. Min-Ho Suh, T.Y.L. Robust Optimal Design of Wastewater Reuse Network of Plating Process. J. Chem. Eng. Jpn. 2002, 35, 863-873. doi:10.1252/jcej.35.863. [CrossRef]

33. Koppol, A.P.R.; Bagajewicz, M.J. Financial Risk Management in the Design of Water Utilization Systems in Process Plants. Ind. Eng. Chem. Res. 2003, 42, 5249-5255. doi:10.1021/ie0203882. [CrossRef]

34. Tan, R.R.; Cruz, D.E. Synthesis of robust water reuse networks for single-component retrofit problems using symmetric fuzzy linear programming. Comput. Chem. Eng. 2004, 28, 2547-2551. doi:10.1016/j.compchemeng.2004.06.016. [CrossRef]

35. Karuppiah, R.; Grossmann, I.E. Global optimization of multiscenario mixed integer nonlinear programming models arising in the synthesis of integrated water networks under uncertainty. Comput. Chem. Eng. 2008, 32, 145-160. doi:10.1016/j.compchemeng.2007.03.007. [CrossRef]

36. Chew, I.M.L.; Tan, R.; Ng, D.K.S.; Foo, D.C.Y.; Majozi, T.; Gouws, J. Synthesis of Direct and Indirect Interplant Water Network. Ind. Eng. Chem. Res. 2008, 47, 9485-9496. doi:10.1021/ie800072r. [CrossRef]

37. Zhou, R.J.; Li, L.J.; Dong, H.G.; Grossmann, I.E. Synthesis of Interplant Water-Allocation and Heat-Exchange Networks. Part 1: Fixed Flow Rate Processes. Ind. Eng. Chem. Res. 2012, 51, 4299-4312. doi:10.1021/ie2014789. [CrossRef]

38. Zhou, R.J.; Li, L.J. Simultaneous Optimization of Property-Based Water-Allocation and Heat-Exchange Networks with State-Space Superstructure. Ind. Eng. Chem. Res. 2015, 54, 9758-9769. doi:10.1021/acs.iecr.5b01486. [CrossRef]

39. Ibrić, N.; Ahmetović, E.; Kravanja, Z.; Maréchal, F.; Kermani, M. Synthesis of single and interplant non-isothermal water networks. J. Environ. Manag. 2017, 203, 1095-1117. doi:10.1016/j.jenvman.2017.05.001. [CrossRef] [PubMed]

40. Kermani, M.; Wallerand, A.S.; Kantor, I.D.; Maréchal, F. A Hybrid Methodology for Combined Interplant Heat, Water, and Power Integration. In Computer Aided Chemical Engineering; Espuña, A., Graells, M., Puigjaner, L., Eds.; Elsevier: New York, NY, USA, 2017; Volume 40, pp. 1969-1974.

41. Liew, P.Y.; Theo, W.L.; Wan Alwi, S.R.; Lim, J.S.; Abdul Manan, Z.; Klemeš, J.J.; Varbanov, P.S. Total Site Heat Integration planning and design for industrial, urban and renewable systems. Renew. Sustain. Energy Rev. 2017, 68, Part 2, 964-985. doi:10.1016/j.rser.2016.05.086. [CrossRef]

42. Du, J.; Meng, X.; Du, H.; Yu, H.; Fan, X.; Yao, P. Optimal Design of Water Utilization Network with Energy Integration in Process Industries. Chin. J. Chem. Eng. 2004, 12, 247-255.

43. Renard, L.; Périn-Levasseur, Z.; Salgueiro, L.; Savulescu, L.E.; Maréchal, F.; Benali, M. Combined heat and mass integration: A benchmarking case study. In Proceeding of 22nd European Symposium on Computer Aided Process Engineering; Lockhart Bogle, I.D.; Fairweather, M., Eds.; Elsevier: New York, NY, USA, 2012. 
44. Kermani, M.; Périn-Levasseur, Z.; Benali, M.; Savulescu, L.; Maréchal, F. An Improved Linear Programming Approach for Simultaneous Optimization of Water and Energy. In Computer Aided Chemical Engineering; Klemeš, J.J., Varbanov, P.S., Liew, P.Y., Eds.; Elsevier: New York, NY, USA, 2014; Volume 33, pp. 1561-1566.

45. Ibrić, N.; Ahmetović, E.; Kravanja, Z.; Maréchal, F.; Kermani, M. Simultaneous synthesis of non-isothermal water networks integrated with process streams. Energy 2017, 141, 2587-2612. doi:10.1016/j.energy.2017.07.018. [CrossRef]

46. Savulescu, L.; Kim, J.K.; Smith, R. Studies on simultaneous energy and water minimisation—Part I: Systems with no water re-use. Chem. Eng. Sci. 2005, 60, 3279-3290. doi:10.1016/j.ces.2004.12.037. [CrossRef]

47. Savulescu, L.; Kim, J.K.; Smith, R. Studies on simultaneous energy and water minimisation-Part II: Systems with maximum re-use of water. Chem. Eng. Sci. 2005, 60, 3291-3308. doi:10.1016/j.ces.2004.12.036. [CrossRef]

48. Manan, Z.A.; Tea, S.Y.; Alwi, S.R.W. A new technique for simultaneous water and energy minimisation in process plant. Chem. Eng. Res. Des. 2009, 87, 1509-1519. doi:10.1016/j.cherd.2009.04.013. [CrossRef]

49. Leewongtanawit, B.; Kim, J.K. Improving energy recovery for water minimisation. Energy 2009, 34, 880-893. doi:10.1016/j.energy.2009.03.004. [CrossRef]

50. Wan Alwi, S.R.; Ismail, A.; Manan, Z.A.; Handani, Z.B. A new graphical approach for simultaneous mass and energy minimisation. Appl. Therm. Eng. 2011, 31, 1021-1030. doi:10.1016/j.applthermaleng.2010.11.026. [CrossRef]

51. Ismail, A.; Alwi, S.; Manan, Z. Simultaneous targeting and design for non-mass transfer based water and energy reduction. In Proceedings of the 2011 4th International Conference on Modeling, Simulation and Applied Optimization (ICMSAO), Kuala Lumpur, Malaysia, 19-21 April 2011; pp. 1-8.

52. Martínez-Patiño, J.; Picón-Núñez, M.; Serra, L.M.; Verda, V. Design of water and energy networks using temperature-concentration diagrams. Energy 2011, 36, 3888-3896. doi:10.1016/j.energy.2010.12.042. [CrossRef]

53. Martínez-Patiño, J.; Picón-Núñez, M.; Serra, L.M.; Verda, V. Systematic approach for the synthesis of water and energy networks. Appl. Therm. Eng. 2012, 48, 458-464. doi:10.1016/j.applthermaleng.2011.12.030. [CrossRef]

54. Liao, Z.; Hong, X.; Jiang, B.; Wang, J.; Yang, Y. Novel graphical tool for the design of the heat integrated water allocation networks. AIChE J. 2015, 62, 670-686. doi:10.1002/aic.15049. [CrossRef]

55. Hou, Y.; Wang, J.; Chen, Z.; Li, X.; Zhang, J. Simultaneous integration of water and energy on conceptual methodology for both single- and multi-contaminant problems. Chem. Eng. Sci. 2014, 117, 436-444. doi:10.1016/j.ces.2014.07.004. [CrossRef]

56. Hou, Y.; Xie, W.; Duan, Z.; Wang, J. A conceptual methodology for simultaneous optimization of water and heat with non-isothermal mixing. Front. Chem. Sci. Eng. 2017, 11, 154-165. doi:10.1007/s11705-016-1593-z. [CrossRef]

57. Xie, W.; Hou, Y.; Wang, Y.; Wang, J. A heuristic approach based on a single-temperature-peak design principle for simultaneous optimization of water and energy in fixed flowrate systems. Chem. Eng. Sci. 2016, 152, 323-342. doi:10.1016/j.ces.2016.06.018. [CrossRef]

58. Feng, X.; Li, Y.; Yu, X. Improving Energy Performance of Water Allocation Networks Through Appropriate Stream Merging. Chin. J. Chem. Eng. 2008, 16, 480-484. doi:10.1016/S1004-9541(08)60109-3. [CrossRef]

59. Ataei, A.; Panjeshahi, M.; Karbassian, S. Simultaneous Energy and Water Minimization-Approach for Systems with Optimum Regeneration of Wastewater. Res. J. Environ. Sci. 2009, 3, 604-618. doi:10.3923/rjes.2009.604.618. [CrossRef]

60. Ataei, A.; Yoo, C.K. Simultaneous Energy and Water Optimization in Multiple- Contaminant Systems with Flowrate Changes Consideration. Int. J. Environ. Res. 2010, 4, 11-26.

61. Sahu, G.C.; Bandyopadhyay, S. Energy optimization in heat integrated water allocation networks. Chem. Eng. Sci. 2012, 69, 352-364. doi:10.1016/j.ces.2011.10.054. [CrossRef]

62. Tan, Y.L.; Ng, D.K.S.; El-Halwagi, M.M.; Foo, D.C.Y.; Samyudia, Y. Synthesis of Heat Integrated Resource Conservation Networks with Varying Operating Parameters. Ind. Eng. Chem. Res. 2013, 52, 7196-7210. doi:10.1021/ie302485y. [CrossRef]

63. Liu, L.; Du, J.; El-Halwagi, M.M.; Ponce-Ortega, J.M.; Yao, P. A systematic approach for synthesizing combined mass and heat exchange networks. Comput. Chem. Eng. 2013, 53, 1-13. doi:10.1016/j.compchemeng.2013.02.005. [CrossRef] 
64. De-León Almaraz, S.; Boix, M.; Montastruc, L.; Azzaro-Pantel, C.; Liao, Z.; Domenech, S. Design of a water allocation and energy network for multi-contaminant problems using multi-objective optimization. Process Saf. Environ. Prot. 2016, 108, 348-364. doi:10.1016/j.psep.2016.03.015. [CrossRef]

65. Wang, Y.; Li, R.; Feng, X. Rule-based optimization strategy for energy efficient water networks. Appl. Therm. Eng. 2017, 110, 730-736. doi:10.1016/j.applthermaleng.2016.08.212. [CrossRef]

66. Polley, G.T.; Picón-Núñez, M.; de Jesús López-Maciel, J. Design of water and heat recovery networks for the simultaneous minimisation of water and energy consumption. Appl. Therm. Eng. 2010, 30, 2290-2299. doi:10.1016/j.applthermaleng.2010.03.031. [CrossRef]

67. Sharma, S.; Rangaiah, G.P. Multi-objective Optimization of Heat Integrated Water Networks in Petroleum Refineries. In Computer Aided Chemical Engineering; Klemeš, J.J., Varbanov, P.S., Liew, P.Y., Eds.; Elsevier, 2014; Volume 33, pp. 1531-1536.

68. Liang, Y.; Hui, C.W. A shortcut model for energy efficient water network synthesis. Appl. Therm. Eng. 2016, 96, 88-91. doi:10.1016/j.applthermaleng.2015.11.020. [CrossRef]

69. Boix, M.; Pibouleau, L.; Montastruc, L.; Azzaro-Pantel, C.; Domenech, S. Minimizing water and energy consumptions in water and heat exchange networks. Appl. Therm. Eng. 2012, 36, 442-455. doi:10.1016/j.applthermaleng.2011.10.062. [CrossRef]

70. Zhao, H.P.; Chan, T.C.; Liu, Z.Y. Design of water and heat networks with single contaminant. Asia-Pac. J. Chem. Eng. 2015, 10, 219-227. doi:10.1002/apj.1866. [CrossRef]

71. Yang, L.; Grossmann, I.E. Water Targeting Models for Simultaneous Flowsheet Optimization. Ind. Eng. Chem. Res. 2013, 52, 3209-3224. doi:10.1021/ie301112r. [CrossRef]

72. Teles, J.P.; Castro, P.M.; Novais, A.Q. MILP-based initialization strategies for the optimal design of water-using networks. Chem. Eng. Sci. 2009, 64, 3736-3752. doi:10.1016/j.ces.2009.04.051. [CrossRef]

73. Castro, P.M.; Matos, H.A.; Novais, A.Q. An efficient heuristic procedure for the optimal design of wastewater treatment systems. Resour. Conserv. Recycl. 2007, 50, 158-185. doi:10.1016/j.resconrec.2006.06.013. [CrossRef]

74. Gundersen, T.; Naess, L. The synthesis of cost optimal heat exchanger networks: An industrial review of the state of the art. Comput. Chem. Eng. 1988, 12, 503-530. doi:10.1016/0098-1354(88)87002-9. [CrossRef]

75. Furman, K.C.; Sahinidis, N.V. A Critical Review and Annotated Bibliography for Heat Exchanger Network Synthesis in the 20th Century. Ind. Eng. Chem. Res. 2002, 41, 2335-2370. doi:10.1021/ie010389e. [CrossRef]

76. Linnhoff, B.; Hindmarsh, E. The pinch design method for heat exchanger networks. Chem. Eng. Sci. 1983, 38, 745-763. doi:10.1016/0009-2509(83)80185-7. [CrossRef]

77. Papoulias, S.A.; Grossmann, I.E. A structural optimization approach in process synthesis-II: Heat recovery networks. Comput. Chem. Eng. 1983, 7, 707-721. doi:10.1016/0098-1354(83)85023-6. [CrossRef]

78. Maréchal, F.; Kalitventzeff, B. Process integration: Selection of the optimal utility system. Comput. Chem. Eng. 1998, 22 (Suppl. 1), S149-S156. doi:10.1016/S0098-1354(98)00049-0. [CrossRef]

79. Marechal, F.; Boursier, I.; Kalitventzeff, B. Synep1 : A methodology for energy integration and optimal heat exchanger network synthesis. Comput. Chem. Eng. 1989, 13, 603-610. doi:10.1016/0098-1354(89)85044-6. [CrossRef]

80. Cerda, J.; Westerburg, A.W. Synthesizing heat exchanger networks having restricted stream/stream matches using transportation problem formulations. Chem. Eng. Sci. 1983, 38, 1723-1740. doi:10.1016/0009-2509(83)85029-5. [CrossRef]

81. Floudas, C.A.; Ciric, A.R.; Grossmann, I.E. Automatic synthesis of optimum heat exchanger network configurations. AIChE J. 1986, 32, 276-290. doi:10.1002/aic.690320215. [CrossRef]

82. Floudas, C.A.; Ciric, A.R. Strategies for overcoming uncertainties in heat exchanger network synthesis. Comput. Chem. Eng. 1989, 13, 1133-1152. doi:10.1016/0098-1354(89)87017-6. [CrossRef]

83. Balas, E.; Jeroslow, R. Canonical Cuts on the Unit Hypercube. SIAM J. Appl. Math. 1972, 23, 61-69. [CrossRef]

84. Ahmad, S. Heat Exchanger Networks: Cost Trade-Offs in Energy and Capital. Ph.D. Thesis, University of Manchester, Institute of Science and Technology, Manchester, UK, 1985.

85. Gundersen, T.; Grossmann, I.E. Improved optimization strategies for automated heat exchanger network synthesis through physical insights. Comput. Chem. Eng. 1990, 14, 925-944. doi:10.1016/0098-1354(90)87050-Y. [CrossRef] 
86. Gundersen, T.; Duvold, S.; Hashemi-Ahmady, A. An extended vertical MILP model for Heat Exchanger Network Synthesis. Comput. Chem. Eng. 1996, 20, S97-S102. doi:10.1016/0098-1354(96)00027-0. [CrossRef]

87. Yee, T.F.; Grossmann, I.E. Simultaneous optimization models for heat integration-II. Heat exchanger network synthesis. Comput. Chem. Eng. 1990, 14, 1165-1184. doi:10.1016/0098-1354(90)85010-8. [CrossRef]

88. Yee, T.F.; Grossmann, I.E.; Kravanja, Z. Simultaneous optimization models for heat integration-I. Area and energy targeting and modeling of multi-stream exchangers. Comput. Chem. Eng. 1990, 14, 1151-1164. doi:10.1016/0098-1354(90)85009-Y. [CrossRef]

89. Yue, D.; Guillén-Gosálbez, G.; You, F. Global optimization of large-scale mixed-integer linear fractional programming problems: A reformulation-linearization method and process scheduling applications. AIChE J. 2013, 59, 4255-4272. doi:10.1002/aic.14185. [CrossRef]

90. Bagajewicz, M.J.; Pham, R.; Manousiouthakis, V. On the state space approach to mass/heat exchanger network design. Chem. Eng. Sci. 1998, 53, 2595-2621. doi:10.1016/S0009-2509(98)00014-1. [CrossRef]

91. Leewongtanawit, B.; Kim, J.K. Synthesis and optimisation of heat-integrated multiple-contaminant water systems. Chem. Eng. Process. Process Intensif. 2008, 47, 670-694. doi:10.1016/j.cep.2006.12.018. [CrossRef]

92. Kuo, W.C.J.; Smith, R. Design of Water-Using Systems Involving Regeneration. Process Saf. Environ. Prot. 1998, 76, 94-114. doi:10.1205/095758298529399. [CrossRef]

93. Kuo, W.C.J.; Smith, R. Designing for the Interactions Between Water-Use and Effluent Treatment. Chem. Eng. Res. Des. 1998, 76, 287-301. doi:10.1205/026387698524938. [CrossRef]

94. Bai, J.; Feng, X.; Deng, C. Graphically Based Optimization of Single-Contaminant Regeneration Reuse Water Systems. Chem. Eng. Res. Des. 2007, 85, 1178-1187. doi:10.1205/cherd06252. [CrossRef]

95. Feng, X.; Bai, J.; Zheng, X. On the use of graphical method to determine the targets of single-contaminant regeneration recycling water systems. Chem. Eng. Sci. 2007, 62, 2127-2138. doi:10.1016/j.ces.2006.12.081. [CrossRef]

96. Hallale, N. A new graphical targeting method for water minimisation. Adv. Environ. Res. 2002, 6, 377-390. doi:10.1016/S1093-0191(01)00116-2. [CrossRef]

97. Quesada, I.; Grossmann, I.E. Global optimization of bilinear process networks with multicomponent flows. Comput. Chem. Eng. 1995, 19, 1219-1242. doi:10.1016/0098-1354(94)00123-5. [CrossRef]

98. McCormick, G.P. Computability of global solutions to factorable nonconvex programs: Part I - Convex underestimating problems. Math. Programm. 1976, 10, 147-175. doi:10.1007/BF01580665. [CrossRef]

99. Floudas, C.A.; Aggarwal, A.; Ciric, A.R. Global optimum search for nonconvex NLP and MINLP problems. Comput. Chem. Eng. 1989, 13, 1117-1132. doi:10.1016/0098-1354(89)87016-4. [CrossRef]

100. Floudas, C.A.; Visweswaran, V. A global optimization algorithm (GOP) for certain classes of nonconvex NLPs-I. Theory. Comput. Chem. Eng. 1990, 14, 1397-1417. doi:10.1016/0098-1354(90)80020-C. [CrossRef]

101. Ahmetović, E.; Grossmann, I.E. Global superstructure optimization for the design of integrated process water networks. AIChE J. 2011, 57, 434-457. doi:10.1002/aic.12276. [CrossRef]

102. Faria, D.C.; Bagajewicz, M.J. Global Optimization of Water Management Problems Using Linear Relaxation and Bound Contraction Methods. Ind. Eng. Chem. Res. 2011, 50, 3738-3753. doi:10.1021/ie101206c. [CrossRef]

103. Faria, D.C.; Bagajewicz, M.J. A new approach for global optimization of a class of MINLP problems with applications to water management and pooling problems. AIChE J. 2012, 58, 2320-2335. doi:10.1002/aic.12754. [CrossRef]

104. Dong, H.G.; Lin, C.Y.; Chang, C.T. Simultaneous optimization approach for integrated water-allocation and heat-exchange networks. Chem. Eng. Sci. 2008, 63, 3664-3678. doi:10.1016/j.ces.2008.04.044. [CrossRef]

105. Bogataj, M.; Bagajewicz, M.J. Synthesis of non-isothermal heat integrated water networks in chemical processes. Comput. Chem. Eng. 2008, 32,3130-3142. doi:10.1016/j.compchemeng.2008.05.006. [CrossRef]

106. Chen, C.L.; Liao, H.L.; Jia, X.P.; Ciou, Y.J.; Lee, J.Y. Synthesis of heat-integrated water-using networks in process plants. J. Taiwan Inst. Chem. Eng. 2010, 41, 512-521. doi:10.1016/j.jtice.2010.04.004. [CrossRef]

107. Savulescu, L.E.; Sorin, M.; Smith, R. Direct and indirect heat transfer in water network systems. Appl. Therm. Eng. 2002, 22, 981-988. doi:10.1016/S1359-4311(02)00015-7. [CrossRef]

108. Boondarik Leewongwanawit, J.K.K. Design and optimisation of combined water and energy systems. Comput. Aided Chem. Eng. 2004, 18, 439-444. doi:10.1016/S1570-7946(04)80139-1. [CrossRef]

109. Sorin, M.; Savulescu, L.E. On Minimization of the Number of Heat Exchangers in Water Networks. Heat Transf. Eng. 2004, 25, 30-38. doi:10.1080/01457630490459120. [CrossRef] 
110. Bogataj, M.; Bagajewicz, M.J. Design of non-isothermal process water networks. In Computer Aided Chemical Engineering; Valentin, P., Agachi, P.Ş., Eds.; Elsevier: New York, NY, USA, 2007; Volume 24, pp. 377-382.

111. Liao, Z.W.; WU, J.; JIANG, B.; WANG, J.; YANG, Y. Design Energy Efficient Water Utilization Systems Allowing Operation Split*. Chin. J. Chem. Eng. 2008, 16, 16-20. doi:10.1016/S1004-9541(08)60028-2. [CrossRef]

112. Xiao, W.; Zhou, R.j.; Dong, H.G.; Meng, N.; Lin, C.Y.; Adi, V.S.K. Simultaneous optimal integration of water utilization and heat exchange networks using holistic mathematical programming. Korean J. Chem. Eng. 2009, 26, 1161-1174. doi:10.1007/s11814-009-0196-5. [CrossRef]

113. Kim, J.; Kim, J.; Kim, J.; Yoo, C.; Moon, I. A simultaneous optimization approach for the design of wastewater and heat exchange networks based on cost estimation. J. Clean. Prod. 2009, 17, 162-171. doi:10.1016/j.jclepro.2008.04.005. [CrossRef]

114. Feng, X.; Li, Y.; Shen, R. A new approach to design energy efficient water allocation networks. Appl. Therm. Eng. 2009, 29, 2302-2307. doi:10.1016/j.applthermaleng.2008.11.007. [CrossRef]

115. Liao, Z.W.; Rong, G.; Wang, J.; Yang, Y. Systematic Optimization of Heat-Integrated Water Allocation Networks. Ind. Eng. Chem. Res. 2011, 50, 6713-6727. doi:10.1021/ie1016392. [CrossRef]

116. George, J.; Sahu, G.C.; Bandyopadhyay, S. Heat Integration in Process Water Networks. Ind. Eng. Chem. Res. 2011, 50, 3695-3704. doi:10.1021/ie101098a. [CrossRef]

117. Bandyopadhyay, S.; Sahu, G.C. Energy targeting in heat integrated water networks with isothermal mixing. In Computer Aided Chemical Engineering; Pistikopoulos, E.N., Georgiadis, M.C., Kokossis, A.C., Eds.; Elsevier: New York, NY, USA, 2011; Volume 29, pp. 1989-1993.

118. Zhou, R.J.; Li, L.J.; Dong, H.G.; Grossmann, I.E. Synthesis of Interplant Water-Allocation and Heat-Exchange Networks. Part 2: Integrations between Fixed Flow Rate and Fixed Contaminant-Load Processes. Ind. Eng. Chem. Res. 2012, 51, 14793-14805. doi:10.1021/ie3019752. [CrossRef]

119. Yiqing, L.; Tingbi, M.; Sucai, L.; Xigang, Y. Studies on the effect of non-isothermal mixing on water-using network's energy performance. Comput. Chem. Eng. 2012, 36, 140-148. doi:10.1016/j.compchemeng.2011.07.007. [CrossRef]

120. Tan, Y.L.; Ng, D.K.S.; El-Halwagi, M.M.; Samyudia, Y.; Foo, D.C.Y. Synthesis of Heat-Integrated Resource Conservation Networks. In Computer Aided Chemical Engineering; Karimi, I.A., Srinivasan, R., Eds.; Elsevier: New York, NY, USA, 2012; Volume 31, pp. 985-989.

121. Ahmetović, E.; Kravanja, Z. Solution strategies for the synthesis of heat-integrated process water networks. Chem. Eng. Trans. 2012, 29, 1015-1020. doi:10.3303/CET1229170. [CrossRef]

122. Rojas-Torres, M.G.; Ponce-Ortega, J.M.; Serna-González, M.; Nápoles-Rivera, F.; El-Halwagi, M.M. Synthesis of Water Networks Involving Temperature-Based Property Operators and Thermal Effects. Ind. Eng. Chem. Res. 2013, 52, 442-461. doi:10.1021/ie301433w. [CrossRef]

123. Li, D. Design of heat integrated water networks with multiple contaminants considering non-isothermal mixing. Chem. Ind. Eng. Process 2013, 32, 238-242.

124. Ibrić, N.; Ahmetović, E.; Kravanja, Z. A Two-Step Solution Strategy for the Synthesis of Pinched and Threshold Heat-Integrated Process Water Networks. Chem. Eng. Trans. 2013, 35, 43-48. doi:10.3303/CET1335007. [CrossRef]

125. Chew, I.M.L.; Foo, D.C.Y.; Bonhivers, J.C.; Stuart, P.; Alva-Argaez, A.; Savulescu, L.E. A model-based approach for simultaneous water and energy reduction in a pulp and paper mill. Appl. Therm. Eng. 2013, 51, 393-400. doi:10.1016/j.applthermaleng.2012.08.070. [CrossRef]

126. Ahmetović, E.; Kravanja, Z. Simultaneous synthesis of process water and heat exchanger networks. Energy 2013, 57, 236-250. doi:10.1016/j.energy.2013.02.061. [CrossRef]

127. Tan, Y.L.; Ng, D.K.S.; Foo, D.C.Y.; El-Halwagi, M.M.; Samyudia, Y. Heat integrated resource conservation networks without mixing prior to heat exchanger networks. J. Clean. Prod. 2014, 71, 128-138. doi:10.1016/j.jclepro.2014.01.014. [CrossRef]

128. Jiménez-Gutiérrez, A.; Lona-Ramírez, J.; Ponce-Ortega, J.M.; El-Halwagi, M. An MINLP model for the simultaneous integration of energy, mass and properties in water networks. Comput. Chem. Eng. 2014, 71, 52-66. doi:10.1016/j.compchemeng.2014.07.008. [CrossRef]

129. Ibrić, N.; Ahmetović, E.; Kravanja, Z. Two-step mathematical programming synthesis of pinched and threshold heat-integrated water networks. J. Clean. Prod. 2014, 77, 116-139. doi:10.1016/j.jclepro.2014.01.004. [CrossRef] 
130. Ibrić, N.; Ahmetović, E.; Kravanja, Z. Synthesis of Water, Wastewater Treatment, and Heat-Exchanger Networks. In Computer Aided Chemical Engineering; Klemeš, J.J., Varbanov, P.S., Liew, P.Y., Eds.; Elsevier: New York, NY, USA, 2014; VolUME 33, pp. 1843-1848.

131. Ibrić, N.; Ahmetović, E.; Kravanja, Z. Simultaneous optimization of water and energy within integrated water networks. Appl. Therm. Eng. 2014, 70, 1097-1122. doi:10.1016/j.applthermaleng.2014.03.019. [CrossRef]

132. Chen, Z.; Hou, Y.; Li, X.; Wang, J. Simultaneous optimization of water and heat exchange networks. Korean J. Chem. Eng. 2014, 31, 558-567. doi:10.1007/s11814-013-0236-z. [CrossRef]

133. Ahmetović, E.; Kravanja, Z. Simultaneous optimization of heat-integrated water networks involving process-to-process streams for heat integration. Appl. Therm. Eng. 2014, 62, 302-317. doi:10.1016/j.applthermaleng.2013.06.010. [CrossRef]

134. Ahmetović, E.; Ibrić, N.; Kravanja, Z. Optimal design for heat-integrated water-using and wastewater treatment networks. Appl. Energy 2014, 135, 791-808. doi:10.1016/j.apenergy.2014.04.063. [CrossRef]

135. Zhou, L.; Liao, Z.; Wang, J.; Jiang, B.; Yang, Y.; Yu, H. Simultaneous Optimization of Heat-Integrated Water Allocation Networks Using the Mathematical Model with Equilibrium Constraints Strategy. Ind. Eng. Chem. Res. 2015, 54, 3355-3366. doi:10.1021/ie501960e. [CrossRef]

136. Liu, Z.; Luo, Y.; Yuan, X. Synthesis of heat-integrated water allocation network considering non-isothermal mixing. CIESC J. 2014, 65, 285-291. doi:10.3969/j.issn.0438-1157.2014.01.037. [CrossRef]

137. Liu, Z.; Luo, Y.; Yuan, X. Simultaneous integration of water and energy in heat-integrated water allocation networks. AIChE J. 2015, 61, 2202-2214. doi:10.1002/aic.14823. [CrossRef]

138. Ghazouani, S.; Zoughaib, A.; Pelloux-Prayer, S. Simultaneous heat integrated resource allocation network targeting for total annual cost considering non-isothermal mixing. Chem. Eng. Sci. 2015, 134, 385-398. doi:10.1016/j.ces.2015.05.027. [CrossRef]

139. Yan, F.; Wu, H.; Li, W.; Zhang, J. Simultaneous optimization of heat-integrated water networks by a nonlinear program. Chem. Eng. Sci. 2016, 140, 76-89. doi:10.1016/j.ces.2015.09.036. [CrossRef]

140. Torkfar, F.; Avami, A. A simultaneous methodology for the optimal design of integrated water and energy networks considering pressure drops in process industries. Process Saf. Environ. Prot. 2016. doi:10.1016/j.psep.2016.06.008. [CrossRef]

141. Jagannath, A.; Almansoori, A. Sequential synthesis of heat integrated water networks: A new approach and its application to small and medium sized examples. Comput. Chem. Eng. 2016, 90, 44-61. doi:10.1016/j.compchemeng.2016.04.016. [CrossRef]

142. Ibrić, N.; Ahmetović, E.; Kravanja, Z. Mathematical programming synthesis of non-isothermal water networks by using a compact/reduced superstructure and an MINLP model. Clean Technol. Environ. Policy 2016, pp. 1-35. doi:10.1007/s10098-016-1152-9. [CrossRef]

143. Hong, X.; Liao, Z.; Jiang, B.; Wang, J.; Yang, Y. Simultaneous optimization of heat-integrated water allocation networks. Appl. Energy 2016, 169, 395-407. doi:10.1016/j.apenergy.2016.01.059. [CrossRef]

144. Ghazouani, S.; Zoughaib, A.; Bourdiec, S.L. An MILP model for simultaneous mass allocation and heat exchange networks design. Chem. Eng. Sci. 2017, 158, 411-428. doi:10.1016/j.ces.2016.10.041. [CrossRef]

145. Hong, X.; Liao, Z.; Jiang, B.; Wang, J.; Yang, Y. Targeting of heat integrated water allocation networks by one-step MILP formulation. Appl. Energy 2017, 197, 254-269. doi:10.1016/j.apenergy.2017.04.003. [CrossRef]

146. Hong, X.; Liao, Z.; Sun, J.; Jiang, B.; Wang, J.; Yang, Y. Energy and water management for industrial large-scale water networks: a systematic simultaneous optimization approach. ACS Sustain. Chem. Eng. 2017. doi:10.1021/acssuschemeng.7b03740. [CrossRef]

147. Liu, L.; Song, H.; Zhang, L.; Du, J. Heat-integrated water allocation network synthesis for industrial parks with sequential and simultaneous design. Comput. Chem. Eng. 2018, 108, 408-424. doi:10.1016/j.compchemeng.2017.10.002. [CrossRef]

148. Geoffrion, A.M. Generalized Benders decomposition. J. Optim. Theory Appl. 1972, 10, $237-260$. doi:10.1007/BF00934810. [CrossRef]

149. Grossmann, I.E.; Viswanathan, J.; Vecchietti, A.; Raman, R.; Kalvelagen, E. Gams/dicopt: A discrete continuous optimization package. Available online: http://www.amsterdamoptimization.com/pdf/ dicopt.pdf (accessed on 6 April 2018) . 
150. Sahinidis, N.V. BARON 17.8.9: Global Optimization of Mixed-Integer Nonlinear Programs, User's Manual; The Optimization Firm, LLC: Pittsburgh, PA, USA, 2017.

151. Tawarmalani, M.; Sahinidis, N.V. A polyhedral branch-and-cut approach to global optimization. Math. Programm. 2005, 103, 225-249. doi:10.1007/s10107-005-0581-8. [CrossRef]

152. Lin, Y.; Schrage, L. The Global Solver in the LINDO API. Optim. Methods Softw. 2009, 24, $657-668$. doi:10.1080/10556780902753221. [CrossRef]

153. Duran, M.A.; Grossmann, I.E. An outer-approximation algorithm for a class of mixed-integer nonlinear programs. Math. Programm. 1986, 36, 307-339. doi:10.1007/BF02592064. [CrossRef]

154. Xiao, W.; Dong, H.; Li, X.; Yao, P.; Luo, X.; Wilfried, R. Synthesis of Large-scale Multistream Heat Exchanger Networks Based on Stream Pseudo Temperature. Chin. J. Chem. Eng. 2006, 14, 574-583. doi:10.1016/S1004-9541(06)60118-3. [CrossRef]

155. Baumrucker, B.T.; Renfro, J.G.; Biegler, L.T. MPEC problem formulations and solution strategies with chemical engineering applications. Comput. Chem. Eng. 2008, 32, 2903-2913. doi:10.1016/j.compchemeng.2008.02.010. [CrossRef]

156. Pham, V.; Laird, C.; El-Halwagi, M. Convex Hull Discretization Approach to the Global Optimization of Pooling Problems. Ind. Eng. Chem. Res. 2009, 48, 1973-1979. doi:10.1021/ie8003573. [CrossRef]

157. Papalexandri, K.; Pistikopoulos, E.; Floudas, A. Mass exchange networks for waste minimization: A simultaneous approach. Chem. Eng. Res. Des. 1994, 72, 279-294.

158. Luo, Y.; Liu, Z.; Luo, S.; Yuan, X. Thermodynamic analysis of non-isothermal mixing's influence on the energy target of water-using networks. Comput. Chem. Eng. 2014, 61, 1-8. doi:10.1016/j.compchemeng.2013.10.008. [CrossRef]

159. Martinez-Patiño, J.; Serra, L.; Verda, V.; Picón-Núñez, M.; Rubio-Maya, C. Thermodynamic Analysis of Simultaneous Heat and Mass Transfer Systems. J. Energy Resour. Technol. 2016, 138, 062006. doi:10.1115/1.4034068. [CrossRef]

160. Gleick, P.H. Water and Energy. Annu. Rev. Energy Environ. 1994, 19, $267-299$. doi:10.1146/annurev.eg.19.110194.001411. [CrossRef]

161. Garcia, D.J.; You, F. The water-energy-food nexus and process systems engineering: A new focus. Comput. Chem. Eng. 2016, 91, 49-67. doi:10.1016/j.compchemeng.2016.03.003. [CrossRef]

162. Martinez-Hernandez, E.; Samsatli, S. Biorefineries and the food, energy, water nexus-towards a whole systems approach to design and planning. Curr. Opin. Chem. Eng. 2017, 18, 16-22. doi:10.1016/j.coche.2017.08.003. [CrossRef]

163. Lee, M.; Keller, A.A.; Chiang, P.C.; Den, W.; Wang, H.; Hou, C.H.; Wu, J.; Wang, X.; Yan, J. Water-energy nexus for urban water systems: A comparative review on energy intensity and environmental impacts in relation to global water risks. Appl. Energy 2017, 205, 589-601. doi:10.1016/j.apenergy.2017.08.002. [CrossRef]

164. Albrecht, T.R.; Crootof, A.; Scott, C.A. The Water-Energy-Food Nexus: A systematic review of methods for nexus assessment. Environ. Res. Lett. 2018, 13, 043002. doi:10.1088/1748-9326/aaa9c6. [CrossRef]

165. Dai, J.; Wu, S.; Han, G.; Weinberg, J.; Xie, X.; Wu, X.; Song, X.; Jia, B.; Xue, W.; Yang, Q. Water-energy nexus: A review of methods and tools for macro-assessment. Appl. Energy 2018, 210, 393-408. doi:10.1016/j.apenergy.2017.08.243. [CrossRef]

166. Inselberg, A. The plane with parallel coordinates. Vis. Comput. 1985, 1, 69-91. doi:10.1007/BF01898350. [CrossRef]

167. Mao, T.; Luo, Y.; Yuan, X. Design method of heat integrated water networks considering non-isothermal mixing. CIESC J. 2010, 61, 369-377.

(C) 2018 by the authors. Licensee MDPI, Basel, Switzerland. This article is an open access article distributed under the terms and conditions of the Creative Commons Attribution (CC BY) license (http:/ / creativecommons.org/licenses/by/4.0/). 